\title{
Homogeneous nucleation of carbon dioxide in supersonic
} nozzles I : experiments and classical theories

\section{Dingilian, Kayane K.}

2020-09-14

Dingilian , K K , Halonen , R , Tikkanen, V , Reischl , B , Vehkamaki , H \& Wyslouzil , B E 2020 , ' Homogeneous nucleation of carbon dioxide in supersonic nozzles I : experiments and classical theories ', Physical Chemistry Chemical Physics, vol. 22 , no. 34 , pp. 19282-19298 . https://doi.org/10.1039/d0cp02279a

http://hdl.handle.net/10138/332972

https://doi.org/10.1039/d0cp02279a

acceptedVersion

Downloaded from Helda, University of Helsinki institutional repository.

This is an electronic reprint of the original article.

This reprint may differ from the original in pagination and typographic detail.

Please cite the original version. 


\title{
Homogeneous nucleation of carbon dioxide in supersonic nozzles I: Experiments and Classical Theories
}

\author{
Kayane K. Dingilian, ${ }^{1}$ Roope Halonen, ${ }^{2}$ Valtteri Tikkanen, ${ }^{2}$ Bernhard Reischl, ${ }^{2}$ Hanna \\ Vehkamäki, ${ }^{2}$ and Barbara E. Wyslouzil ${ }^{1,3}$ \\ ${ }^{1}$ William G. Lowrie Department of Chemical and Biomolecular Engineering, The Ohio State University, \\ Columbus, Ohio 43210, USA \\ ${ }^{2}$ Institute for Atmospheric and Earth System Research / Physics, University of Helsinki, P.O. Box 64, FI- \\ 00014, Helsinki, Finland \\ ${ }^{3}$ Department of Chemistry and Biochemistry, The Ohio State University, Columbus, Ohio 43210, USA
}

We studied the homogeneous nucleation of carbon dioxide in the carrier gas argon for concentrations of $\mathrm{CO}_{2}$ ranging from 2 to 39 mole percent using three experimental methods. Position-resolved pressure trace measurements (PTM) determined that the onset of nucleation occurred at temperatures between 75 and $92 \mathrm{~K}$ with corresponding $\mathrm{CO}_{2}$ partial pressures of 39 to $793 \mathrm{~Pa}$. Small angle X-ray scattering (SAXS) measurements provided particle size distributions and aerosol number densities. Number densities of approximately $10^{12} \mathrm{~cm}^{-3}$, and characteristic times ranging from 6 to $13 \mu \mathrm{s}$, resulted in measured nucleation rates on the order of $5 \times 10^{17}$ $\mathrm{cm}^{-3} \mathrm{~s}^{-1}$, values that are consistent with other nucleation rate measurements in supersonic nozzles. Finally, we used Fourier transform infrared (FTIR) spectroscopy to identify that the condensed $\mathrm{CO}_{2}$ particles were crystalline cubic solids with either sharp or rounded corners. Molecular dynamics simulations, however, suggest that $\mathrm{CO}_{2}$ forms liquid-like critical clusters before transitioning to the solid phase. Furthermore, the critical clusters are not in thermal equilibrium with the carrier gas. Comparisons with nucleation theories were therefore made assuming liquidlike critical clusters and incorporating non-isothermal correction factors. 


\section{INTRODUCTION}

Carbon capture and storage (CCS) technologies aim to reduce carbon dioxide $\left(\mathrm{CO}_{2}\right)$ emissions to the atmosphere. Leading CCS methods include physical and chemical absorption, cryogenic separation (Joule-Thomson valve or distillation), and membrane separation. ${ }^{1-5}$ Although these methods are efficient in preventing $\mathrm{CO}_{2}$ release, they can require complex machinery and/or chemicals that negatively impact the environment. In particular, absorption in monoethanolamine (MEA), requires significant amounts of energy to recover the $\mathrm{CO}_{2}$ and regenerate the absorbent. ${ }^{6}$ Furthermore, fugitive emissions of MEA can react with radicals present in the atmosphere to form hazardous compounds such as nitramine and nitrosamine, ${ }^{7,8}$ as well as combine with sulfuric acid to produce new fine particles in the atmosphere. ${ }^{9}$

Condensation from supersonic flows has been studied in the laboratory ${ }^{10-17}$ for over 90 years, and more recently, supersonic separators have been developed to remove condensable materials from natural gas. ${ }^{18-21,22,23}$ In both cases, a gas mixture expands and cools as it flows through the nozzle, promoting the transition of condensable materials from the vapor to the condensed phase. In a separator, the droplets or particles are then removed from the gas via inertial separation. Advantages of supersonic separation over other cryogenic approaches include lower power usage, a higher temperature drop, and greater system pressure recovery. ${ }^{21}$ A major challenge, however, is ensuring that condensate particles are large enough (generally greater than $1 \mu \mathrm{m}$ in diameter) for inertial separation to be effective. The success of these devices in separating hydrocarbons from natural gas ${ }^{21}$ has led to interest in applying supersonic separators to the removal of other compounds, in particular $\mathrm{CO}_{2}$.

A number of research groups have modeled the condensation of carbon dioxide in supersonic nozzles for use in CCS. ${ }^{12,13,17,24-27}$ Even though these groups claim to achieve significant $\mathrm{CO}_{2}$ separation, many use assumptions that are not reasonable, for example assuming that condensation starts as soon as the saturation ratio of $\mathrm{CO}_{2}$ vapor, $S$, is greater than one. ${ }^{12}$ Here $S=p_{\mathrm{v}} / p_{\mathrm{ve}}(T)$ where $p_{\mathrm{v}}$ is partial pressure of $\mathrm{CO} 2$ and $p_{\mathrm{ve}}(T)$ is the equilibrium vapor pressure at temperature $T$. Accurately modeling nucleation and particle growth is critical for designing efficient supersonic separators, since these processes determine the final particle size distributions and, therefore, whether inertial separation is possible. To our knowledge, $\mathrm{CO}_{2}$ 
nucleation rates or growth rates have only been quantified experimentally in post nozzle flows ${ }^{28}$ that correspond to rather extreme saturation ratios, i.e. $\ln (S) \sim 150$.

The earliest experimental studies of condensation in supersonic nozzles were performed on nitrogen, humid air, and steam. ${ }^{29-31}$ Duff ${ }^{14}$ made the first $\mathrm{CO}_{2}$ measurements and characterized conditions at the onset of condensation for temperatures ranging from $\sim 160-180 \mathrm{~K}$ and pressures between $\sim 128-328 \mathrm{kPa}$, i.e. in the region of the phase diagram where solid is the stable phase but the liquid is supercooled by only $28-37 \mathrm{~K}$. Recently, Lettieri et al. ${ }^{11}$ performed similar experiments but expansions started from the supercritical state, and condensation occurred under conditions where the liquid is the stable phase. Finally, Lippe et al. ${ }^{28}$ followed $\mathrm{CO}_{2}$ cluster formation and growth in the post-nozzle flow of a Laval expansion using both mass spectrometry and Infrared absorption spectroscopy. Clusters appeared at $\mathrm{CO}_{2}$ partial pressures of 0.04 and $0.065 \mathrm{~Pa}$ and temperatures of $\sim 31$ and $34 \mathrm{~K}$, i.e. at extreme saturation ratios with respect to the solid $(\ln (S) \sim 150)$ or liquid. In related work, Tanimura et al. ${ }^{10}$ characterized the onset of heterogeneous nucleation of carbon dioxide on water ice particles in supersonic nozzles, and Park and Wyslouzil ${ }^{32}$ examined heterogeneous nucleation of $\mathrm{CO}_{2}$ on $n$-alkane particles. Both found that in the presence of particles, heterogeneous condensation starts close to the extrapolated $\mathrm{CO}_{2}$ vapor-liquid equilibrium line. In summary, despite a number of experimental efforts, quantitative experimental nucleation rate measurements for $\mathrm{CO}_{2}$ in any experimental device remain rare.

Experimental nucleation rates are most often compared to the predictions of classical nucleation theory and its variants. These expressions are convenient because they depend only on the properties of the bulk nucleating phase. Unfortunately, quantitative agreement between experimental rates and the predictions of classical theory is frequently rather poor. The lack of agreement is often blamed on (1) the capillarity assumption and (2) the frequent need to extrapolate physical properties to temperatures beyond the range for which measurements exist. Computer simulations provide a complementary tool to study nucleation and the early stages of particle growth from the vapor phase. While quantum chemistry methods, canonical Monte Carlo simulations and various free energy calculation techniques are restricted to thermodynamic equilibrium, direct molecular dynamics simulations (MD) can be used to study non-equilibrium processes and cluster growth pathways during nucleation. From MD results, nucleation rates can be calculated using mean-first-passage-time, or threshold methods, ${ }^{33}, 34$ and the effects of non- 
isothermal nucleation ${ }^{35}$ can be investigated directly. Furthermore, the nature of the critical cluster, for example whether it is liquid-like or crystalline, can also be examined.

To date nucleation simulations have focused primarily on Lennard-Jones systems and various water models, ${ }^{36-38}$ with more limited investigations of alkanes, alcohols and metals. ${ }^{39}, 40$ Only Horsch et al. ${ }^{41}$ have performed MD studies of $\mathrm{CO}_{2}$ nucleation, describing intermolecular interactions of the molecule by a two-center Lennard-Jones model with an embedded point quadrupole. Their simulations were carried out well above the triple point and, for the reported value of surface tension, the $\mathrm{CO}_{2}$ nucleation rates from simulation matched the predictions of classical nucleation theory quite well. The simulations were conducted without any carrier gas and isokinetic scaling enforced constant temperature on the system. More recent studies, ${ }^{34,35,42-44}$ suggest that this approach is not suitable for directly simulating a nucleating vapor because it removes heat from the nucleating clusters in an unphysical manner. To the best of our knowledge, there are no other atomistic simulations of the homogeneous nucleation of $\mathrm{CO}_{2}$ from the vapor phase.

In this paper, the first in a series, we focus on the results of our experimental studies of $\mathrm{CO}_{2}$ nucleation in supersonic nozzles at temperatures of $\sim 75-90 \mathrm{~K}$ and partial pressures between $\sim 70$ and $800 \mathrm{~Pa}$. For carbon dioxide-argon mixtures containing from 2-39 $\mathrm{mol}^{\circ} \mathrm{CO}_{2}$, we characterized the position-resolved flow properties using pressure trace measurements and followed changes in the aerosol size distribution using small angle $\mathrm{X}$-ray scattering. We also performed limited Fourier transform infrared spectroscopy experiments to characterize the nature of the condensed phase. For solid $\mathrm{CO}_{2}$, this technique is also sensitive to particle shape ${ }^{45} \mathrm{We}$ compare our results to a number of variants of classical nucleation theory and scaling laws, because understanding the accuracy of these approaches is important for adequately modeling separation devices. A detailed report on the fully atomistic molecular dynamics simulations of the nucleation of $\mathrm{CO}_{2}$ in an argon atmosphere, at mixing ranges and temperatures similar to those in the experiments, will be available in the second paper of this series. Here, the simulations simply provide insight into the structure of the critical and slightly post-critical clusters to help rationalize the choice of physical properties required in the comparisons with nucleation theory. In addition, based on the results from the nucleation simulations, we are able to improve the liquid drop model used in classical theory by adding a curvature term to the expression for the cluster formation free energy. Finally, we note that in this paper we will use "clusters" to refer to 
$\mathrm{CO}_{2}$ aggregates containing a small, countable number of $\mathrm{CO}_{2}$ molecules, whereas "particles" are the nanoscale objects characterized in the experiments.

The outline of the paper is as follows. Section II summarizes the basis of classical nucleation theory, and Section III details the experimental and simulation methods. Section IV presents detailed results on experimental measurements and the simulation data relevant to this paper. Section V compares the experiment results to theoretical predictions using both classical and scaling approaches. Section VI summarizes the work, and the conclusions we have drawn.

\section{CLASSICAL NUCLEATION THEORY}

In classical nucleation theory, the formation of a new phase from a supersaturated vapor is assumed to proceed by the formation of clusters that grow by monomer addition and decay by monomer loss. Furthermore, the rate at which fragments of the new phase appear depends sensitively on the number density of the critical clusters. The latter correspond to the smallest clusters for which the probability of cluster growth becomes higher than the probability of cluster decay. If the gas phase is ideal, the classical expression for the nucleation rate can be written as

$$
J=f \beta_{n *} \mathbb{Z} S^{n *} N_{n *}^{\mathrm{eq}},
$$

where $n^{*}$ is the number of monomers in the critical cluster, $f$ is the non-isothermal correction factor, $\mathbb{Z}$ is the Zeldovich non-equilibrium factor, $S$ is the saturation ratio, and $N_{n *}^{\text {eq }}$ is the number density of critical clusters at the equilibrium vapor pressure $p_{\mathrm{ve}}$. The collision rate, $\beta_{n}$, between monomers and an $n$-cluster with radii $r_{1}$ and $r_{\mathrm{n}}=r_{1} n^{1 / 3}$, respectively, can be obtained from kinetic gas theory as

$$
\beta_{n}=N_{1} \sqrt{\frac{8 \pi k_{B} T}{\mu}}\left(r_{1}+r_{n}\right)^{2} .
$$

Here, $N_{1}$ is the monomer number density, $k_{B}$ is the Boltzmann constant, $T$ is temperature and $\mu=n m /(n+1)$ is the reduced mass where $m$ is the mass of a monomer. The monomer radius is calculated from the molecular volume $v$ as $r_{1}=(3 v / 4 \pi)^{1 / 3}$.

The cluster number density distribution in the supersaturated vapor can be expressed as ${ }^{46-}$ 48 


$$
N_{n}=S^{n} N_{n}^{e q}=N_{1} \exp \left(-\frac{\Delta W_{n}^{\mathrm{eq}}-\Delta W_{1}^{\mathrm{eq}}-(n-1) k_{B} T \ln S}{k_{B} T}\right)=N_{1} \exp \left(-\frac{\Delta W_{n}}{k_{B} T}\right) .
$$

This construction satisfies both the law of mass action and the requirement for self-consistency. In the liquid drop model, the equilibrium formation free energy is defined as

$$
\Delta W_{n}^{\mathrm{eq}}=A_{n} \sigma,
$$

where $A_{n}$ is the cluster's spherical surface area and $\sigma$ the interfacial surface tension. In the supersaturated vapor, the critical cluster size $n^{*}$ is found by setting the derivative of $\Delta W_{n}$ with respect to $n$ equal to zero to yield

$$
n^{*}=\frac{32 \pi \sigma^{3} v_{l}^{2}}{3(k T \ln S)^{3}}
$$

The corresponding critical cluster radius $r^{*}$ is

$$
r^{*}=\frac{2 v \sigma}{k_{B} T \ln S}
$$

and the Zeldovich factor is

$$
\mathbb{Z}=\frac{v}{2 \pi r^{* 2}} \sqrt{\frac{\sigma}{k_{B} T}} .
$$

Finally, in the current experiments the high $\mathrm{CO}_{2}$ concentrations may lead to critical clusters that are not in thermal equilibrium with the surrounding gas. We therefore incorporated the classical non-isothermal correction factor of Feder et al. ${ }^{49}$

$$
f=\frac{b^{2}}{b^{2}+q^{2}}
$$

where $b^{2}$ is the mean square of the energy fluctuation of the colliding monomers and carrier gas atoms, labelled with subscript c, given by

$$
b^{2}=2\left(k_{B} T\right)^{2}\left(1+\frac{N_{\mathrm{c}}}{N_{1}} \sqrt{\frac{m}{m_{\mathrm{c}}}}\right),
$$

and $q$ is the energy released upon the addition of a monomer to the cluster,

$$
q=\Delta H_{\mathrm{vap}}-\frac{k_{\mathrm{B}} T}{2}-\frac{\partial A_{n} \sigma}{\partial n}=\Delta H_{\mathrm{vap}}-\frac{k_{\mathrm{B}} T}{2}-k_{\mathrm{B}} T \ln S .
$$


For the $\mathrm{CO}_{2}$-Ar mixtures used in these experiments, $f$ values vary between 0.015 and 0.17 , with the strongest corrections corresponding to the highest $\mathrm{CO}_{2}$ concentrations. The nucleation rates calculated using Eq. (1) - (10), corresponds to the self-consistent version of classical nucleation theory and are denoted by $J_{\mathrm{SCNT}}$.

Classical theory is based on the capillarity approximation which assumes that the surface tension of a cluster equals that of an infinite planar interface, i.e., $\sigma=\sigma_{\infty}$. The surface properties of a cluster containing a relatively small number of monomers can, however, differ significantly from that of a planar surface. The curvature dependency of the surface tension can be expressed using the Tolman equation for a cluster, ${ }^{50}$

$$
\sigma(r)=\frac{\sigma_{\infty}}{1+2 \delta_{\mathrm{T}} / r},
$$

where $\delta_{\mathrm{T}}$ is the Tolman length. Truncating the Taylor expansion after the second term yields

$$
\sigma(r)=\sigma_{\infty}\left(1-\frac{2 \delta_{\mathrm{T}}}{r}\right) .
$$

The difficulty in routinely using this equation is the uncertainty in both the size and sign of the Tolman length. ${ }^{50-52}$

Recent nucleation simulations of the Lennard-Jones fluid, demonstrate that the curvature dependence of $\sigma$ must be included in the formation free energy expression, ${ }^{53}$ and, as we will demonstrate in Paper 2 of this series, the same is true for $\mathrm{CO}_{2}$. Within the framework of standard nucleation kinetics and the liquid droplet model, Tanaka et al. showed that for $\Delta W_{1}=0$, including this correction implies the simulation-based effective Tolman length $\delta_{\mathrm{T}, \mathrm{e}}$ is independent of cluster size and equal to $r_{1} / 2$, where $r_{1}$ is the radius of the monomer. The obtained $\delta_{\mathrm{T}, \mathrm{e}}$ is not directly comparable with explicit values of calculated Tolman lengths which often have negative values. ${ }^{50-52}$ Rather it accounts for various microscopic effects on the surface term in the free energy expression.

Incorporating the simulation-based correction into the classical nucleation theory changes the critical cluster size significantly as these become very small. In particular, the critical cluster radius $r_{\mathrm{ST}}^{*}$ incorporating Tolman theory (Eq. (12)), can be written in terms of the critical cluster radius for classical theory $r^{*}$ as 


$$
r_{\mathrm{ST}}^{*}=\frac{r^{*}}{2}\left(1+\sqrt{1-\frac{2 r_{1}}{r^{*}}}\right) .
$$

Within the context of nucleation, the effect of the correction on the kinetic prefactor $K=f \beta_{n *} \mathbb{Z}$ is insignificant. We denote the nucleation rates calculated using the simulation-based correction factor by $J_{\mathrm{ST}}$.

\section{METHODS AND DATA ANALYSIS}

\section{A. Chemicals and physical properties}

Liquid argon (Ar), 99.998\% purity, was purchased from Praxair in 180L Dewars. Bone dry carbon dioxide $\left(99.9 \% \mathrm{CO}_{2},<10\right.$ ppm water) was purchased from Praxair in compressed gas cylinders.

The relevant physical properties of $\mathrm{Ar}$ and $\mathrm{CO}_{2}$, required to analyze the experimental results and predict nucleation rates, are presented in Table A-1 of the Appendix. Although all physical properties need to be extrapolated in order to reach the experimental temperature range $(\sim 75-90 \mathrm{~K})$, of particular concern are the correlations describing surface tension and density. In Fig. 1(a), we include two surface tension correlations - that proposed by Quinn, ${ }^{54}$ and one we developed based on the Lielmezs-Herrick equation. ${ }^{55}$ The latter has the advantage that it accurately describes data up to the critical point, but the disadvantage that it does not have a finite value at $T=0 \mathrm{~K}$. Over the temperature range of the experiments, however, both equations predict surface tensions that lie well below the surface energy of the solid: Wood estimates surface energy values of 80,92, and $97 \mathrm{mN} / \mathrm{m}$ for the (111), (100), and (110) surfaces of the $\mathrm{CO}_{2}$ crystal respectively, ${ }^{56}$ based on a very simple model using the binding energy per molecule and the number of broken "bonds" at the respective surface. Our molecular dynamics simulations with an atomistic model of $\mathrm{CO}_{2}$ yield surface energies of 169,177 , and $183 \mathrm{mN} / \mathrm{m}$ for the (111), (100), and (110) surfaces, respectively, at $T=75 \mathrm{~K}$. Since the surface tension correlations deviate significantly from each other as the temperature decreases into the range of our experiments, we explore the effects of this uncertainty throughout our analysis. Likewise, as illustrated in Fig. 1(b), the density parametrization for liquid $\mathrm{CO}_{2}$ increases with decreasing temperature and matches that of the solid just below $50 \mathrm{~K}$. 

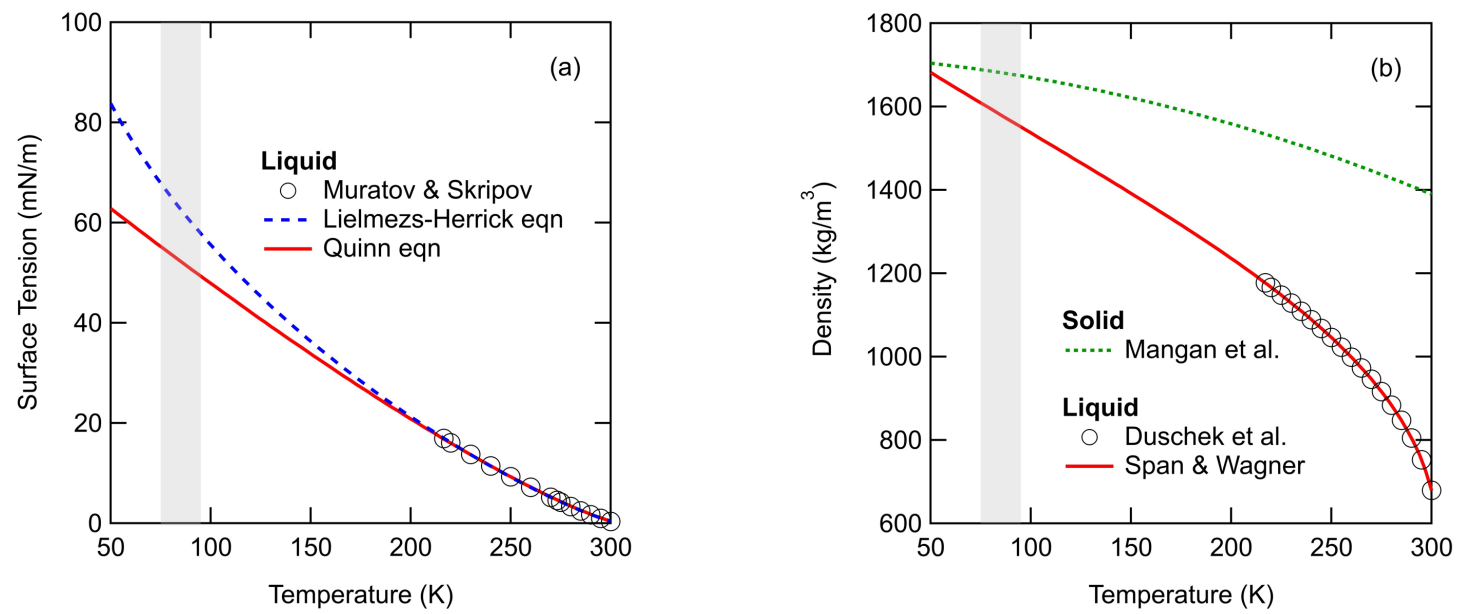

Figure 1: The grey shaded regions indicate the experimental temperature range. (a) Correlations for the planar surface tension of $\mathrm{CO}_{2}$ liquid include that of Quinn ${ }^{54}$ and a new correlation based on the Lielmesz-Herrick ${ }^{55}$ equation and fit to the data of Muratov and Skripov ${ }^{57}$. The parametrization for this equation is outlined in the Supplementary Information. In the temperature range of our experiments, the surface tension estimates deviate significantly. (b) The densities of liquid ${ }^{58,59}$ and $\operatorname{solid}^{60} \mathrm{CO}_{2}$.

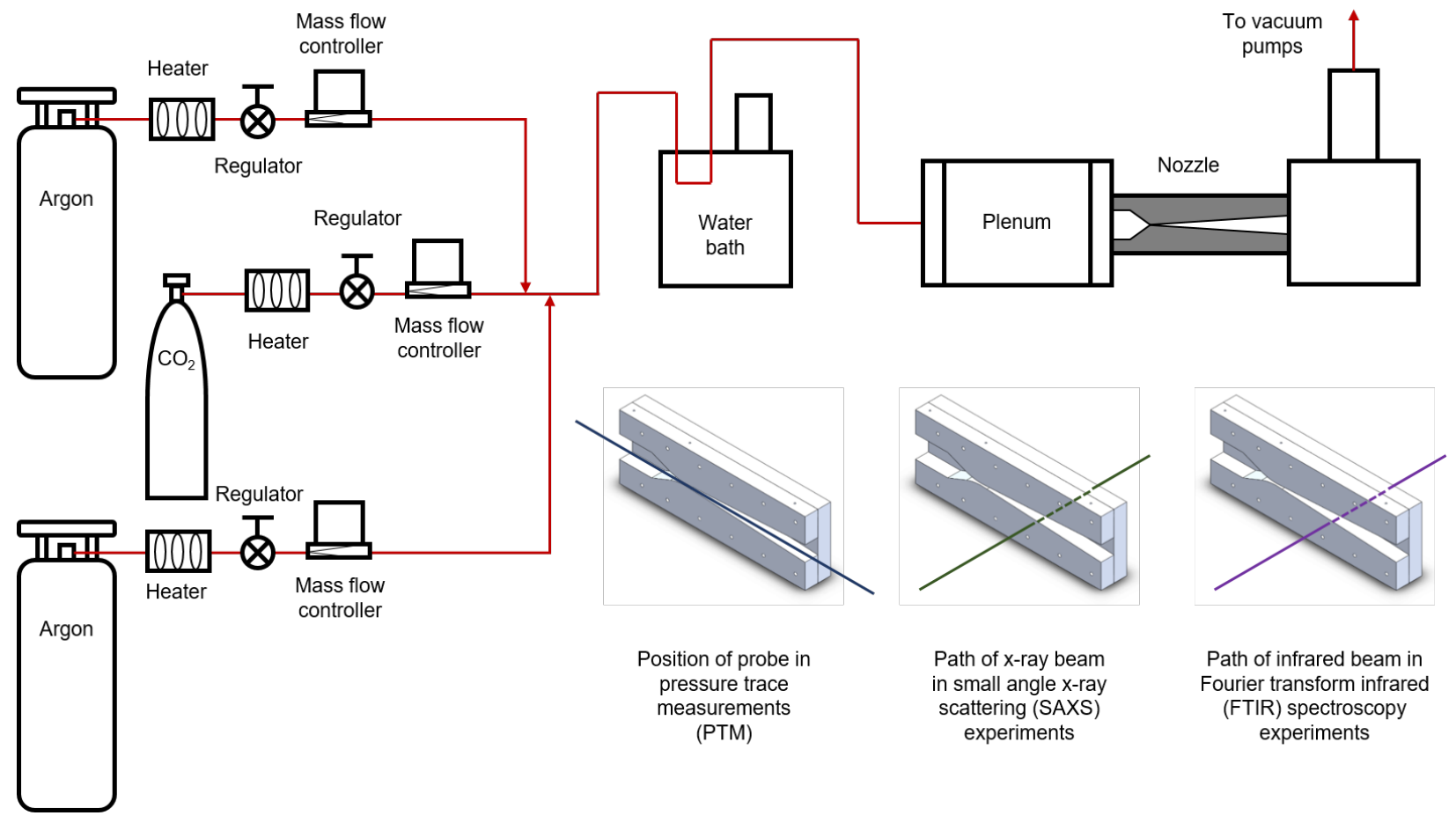

Figure 2. Schematic diagram of the continuous flow supersonic nozzle apparatus. The nozzle cutaway views show the position of the probe or beam in each of the three characterization methods - pressure trace measurements (PTM), small angle x-ray scattering (SAXS), and Fourier transform infrared (FTIR) spectroscopy. Nozzle T3_CaF2 was used for PTM and FTIR experiments, while T3_mica was used for SAXS experiments. 


\section{B. Experimental setup and supersonic nozzle}

A schematic of the continuous flow supersonic nozzle setup is shown in Fig. 2. The carrier gas argon (Ar) is drawn from the gas side of liquid Ar Dewars, brought to room temperature by inline heaters, and its pressure is controlled by regulators. Multiple Dewars are required to ensure a stable flow of room temperature gas. Carbon dioxide $\left(\mathrm{CO}_{2}\right)$ is supplied by a compressed gas cylinder, and its temperature and pressure are also adjusted by an inline heater and regulator. The flow rate of each gas stream is controlled by the appropriate mass flow controller: 300 SLM (MKS 1559) for Ar, 400 or 50 SLM (MKS 1559, MKS GE50A) for $\mathrm{CO}_{2}$. The three gas streams are combined, pass through a static mixer, and the temperature is adjusted and controlled via a circulating water bath. The desired initial conditions are reached in the plenum, where the stagnation temperature is measured using a platinum resistance temperature detector (RTD). All pressures are measured using absolute pressure manometers (MKS 120A Baratron, $0.08 \%$ accuracy of reading). The stagnation pressure $p_{0}$ is determined by correcting a static pressure measurement $p$, made via a sidewall tap upstream of the converging section of the nozzle, using $p_{0}=p+\rho u^{2} / 2$, where $\rho$ is the density of the gas mixture $\left(\mathrm{kg} / \mathrm{m}^{3}\right)$ and $u$ is the velocity. At this point in the flow, $u$ is $\sim 15 \mathrm{~m} / \mathrm{s}$ and the Mach number is $\sim 0.06$. The gas subsequently flows through the nozzle and is exhausted to the atmosphere by two rotary vane vacuum pumps. Prior to each set of experiments, the system was cleaned to minimize background contamination by purging it with Ar at a flow rate of 90 SLM for 1 hour.

Working with carrier gas has a number of advantages in our experiments. In particular, it helps ensure that the effective expansion is relatively independent of the partial pressure of the condensable, and that the gas density is high enough that the flow is well described by the supersonic flow equations. Furthermore, it greatly extends the accessible range of temperatures and $\mathrm{CO}_{2}$ partial pressures that lead to condensation because (1) the heat capacity ratio of Ar is larger than that of $\mathrm{CO}_{2}$ and, thus, lower temperatures are achieved as the concentration of $\mathrm{CO}_{2}$ is reduced, and (2) if the inlet pressure is reduced too far, boundary layers along the nozzle wall grow so quickly that they will merge and destroy the supersonic flow before the desired temperature is reached. Finally, high ratios of carrier gas to condensable minimize nonisothermal nucleation effects, i.e. the decrease in nucleation rates associated with critical clusters that are hotter than the gas phase molecules due to insufficient heat removal. 
Two converging-diverging nozzles with effective expansion rates of $0.385 \mathrm{~cm}^{-1}$ are used in these experiments. For the current experiments, this yields cooling rates of $\sim 4 \mathrm{~K} / \mu \mathrm{s}$ near the throat and $\sim 0.5 \mathrm{~K} / \mu$ s near the onset of condensation. A schematic of the nozzle profile (Nozzle T3) is available in Tanimura et al., ${ }^{10}$ but in the current experiments the width of the nozzle is 12 $\mathrm{mm}$ rather than $6 \mathrm{~mm}$. One nozzle, referred to as nozzle T3_ $\mathrm{CaF}_{2}$, has sidewalls with $3 \mathrm{~mm}$ thick calcium fluoride $\left(\mathrm{CaF}_{2}\right)$ windows and was used for pressure trace measurements (PTM) and Fourier transform infrared (FTIR) measurements. A second nozzle, T3_mica, has sidewalls with $25 \mu \mathrm{m}$ thick mica windows and was used for the small angle X-ray scattering (SAXS) measurements. For all of the characterization techniques, the position $z=0$ and flowtime $t=0$ correspond to the throat.

\section{Pressure trace measurements (PTM)}

When heat is added to a supersonic flow, the pressure increases above that expected for an isentropic expansion of the same gas mixture. Thus, the presence of phase transitions like condensation can be detected quite accurately by static pressure measurements.

Position-resolved static pressure measurements were made along the nozzle centerline using a movable static pressure probe connected to an absolute pressure manometer. Measurements were made from $\sim 1.2 \mathrm{~cm}$ upstream of the throat to $\sim 10.9 \mathrm{~cm}$ downstream of the throat with resolutions of $0.04 \mathrm{~cm}$ near the throat, $0.1 \mathrm{~cm}$ in the regions of high pressure gradient, and $0.2 \mathrm{~cm}$ in the less rapidly changing region further downstream. Two kinds of measurements were performed; dry measurements involving carrier gas only and wet measurements that included the condensable gas, $\mathrm{CO}_{2}$. All experiments started from a stagnation temperature $T_{0}=$ $293.15 \mathrm{~K}\left(20.0^{\circ} \mathrm{C}\right)$ and stagnation pressure $p_{0}=61.15 \pm 0.04 \mathrm{kPa}$. The concentration of $\mathrm{CO}_{2}$ in $\mathrm{Ar}$ varied from 2 to $39 \mathrm{~mol} \%$. The measured variables included the stagnation pressure, stagnation temperature, initial mixture composition, and position resolved static pressure.

We used the wet static pressure data, stagnation conditions, and the area ratio ${ }^{61}$ based on the dry pressure data to obtain position-resolved temperature $T$, density $\rho$, velocity $u$, and condensate mass fraction $g$ of the flow, by integrating the governing equations describing supersonic flow with heat addition. ${ }^{62}$ These include the conservation of mass, momentum, and energy, together with an equation of state. Position-resolved data are converted into timeresolved data using the local flow velocity and the relationship $d t=d z / u$. 


\section{Small angle x-ray scattering (SAXS)}

Small angle X-ray scattering experiments were performed at the 12ID-C beamline at the Advanced Photon Source (APS) at Argonne National Laboratory in Lemont, IL, USA. ${ }^{63}$ Here, the nozzle is mounted on a remotely controlled translation stage and the beam is aligned with the throat using burn paper. The X-ray energy was $12 \mathrm{keV}$, the beam width was $\sim 1 \mathrm{~mm}$, and the sample to detector distance was $2 \mathrm{~m}$. Typically, 10 to 20 single shots with exposure times between 0.7 and $1 \mathrm{~s}$ were made for both the sample and the background. Fixed-position measurements were made $70 \mathrm{~mm}$ downstream of the physical throat where condensation is essentially complete for most experimental conditions. A more limited number of positionresolved measurements were made starting near the end of the nozzle ( $\sim 85 \mathrm{~mm}$ downstream of the throat) and moving upstream until the sample scattering signal was too weak to distinguish it from the background. For these $\mathrm{CO}_{2}$ experiments this corresponds typically to an aerosol with an average particle radius less than $\sim 2 \mathrm{~nm}$.

We used the APS data inversion program to convert 2D scattering patterns to 1D spectra. The absolute scattering intensity was determined via the calibration procedure described by Manka. ${ }^{64}$ The resulting spectra were fit assuming the aerosol followed a Schultz distribution of polydisperse spheres. ${ }^{65}$. From the fit parameters, we calculated the aerosol number density $N$ :

$$
N=\left(\frac{3}{4 \pi}\right)^{2}\left(\frac{(Z+1)^{5}}{(Z+2)(Z+3)(Z+4)(Z+5)(Z+6)}\right)\left(\frac{I_{0}}{\langle r\rangle^{6}\left(\Delta \rho_{S L D}\right)^{2}}\right),
$$

where $\langle r\rangle$ is the average particle radius, $\Delta \rho_{\text {SLD }}$ is the difference in scattering length density between the $\mathrm{CO}_{2}$ particles and the $\mathrm{Ar}-\mathrm{CO}_{2}$ gas mixture, and $\mathrm{Z}$ is given by

$$
Z=\left(\frac{\langle r\rangle}{\delta}\right)^{2}-1
$$

where $\delta$ is the width of the particle size distribution. 


\section{E. Fourier transform infrared (FTIR) spectroscopy}

Fourier transform infrared (FTIR) spectroscopy measurements were performed using the setup described in Park and Wyslouzil. ${ }^{32}$ Briefly, the IR beam exits the side of the PerkinElmer Spectrum 100 and is reflected off a focusing mirror and a plane mirror before it passes through the nozzle. The beam is then reflected off a second focusing mirror onto the MCT detector. The Spectrum 10 software is used to control the spectrometer and produce the spectra. The nozzle is mounted on a moveable base so that measurements can be made as a function of axial position without changing the optical setup. The $\mathrm{CaF}_{2}$ windows and FTIR restrict the wavenumber range to $\sim 1000 \mathrm{~cm}^{-1}$ to $4000 \mathrm{~cm}^{-1}$. For $\mathrm{CO}_{2}$, the most important band in our measurement range is the asymmetric stretch vibrational band ${ }^{66} v_{3}$, located near $2350 \mathrm{~cm}^{-1}$. The spectra were measured using a resolution of $1 \mathrm{~cm}^{-1}$ with apodization turned off to better observe the vapor phase absorption lines.

\section{F. Peak nucleation rate and characteristic time}

The conditions corresponding to the maximum nucleation rate and the characteristic time for nucleation were determined using the methods described by Kim et al. ${ }^{61}$ To summarize, we use nucleation theory, together with the position-resolved temperature and $\mathrm{CO}_{2}$ partial pressure profiles obtained from the pressure trace measurements, to calculate time-resolved nucleation rates, $J_{\text {theory }}(t)$. For most substances ${ }^{61}$ normalizing $J_{\text {theory }}(t)$ by the maximum value, $J_{\text {theory,max }}$, leads to curves (see Fig. 3) whose peak locations and shapes are relatively independent of (1) the exact nucleation rate expression and (2) the assumed temperature dependence of the physical properties of the nucleating substance. Thus, it is reasonable to assume that the conditions that maximize the theoretical nucleation rates are those that maximize the experimental rates. Furthermore, the area under the normalized curve corresponds to the characteristic time associated with the maximum nucleation rate, $\Delta t_{J_{\max }}$, i.e. $\Delta t_{J_{\max }}$ is the time required to nucleate the observed number of particles at the maximum nucleation rate in the absence of coagulation. Values of $\Delta t_{J \max }$ usually vary by less than about $30 \%$ for different nucleation rate expressions, ${ }^{61}$ and in our nozzles typically range between $5-30 \mu \mathrm{s}$. 


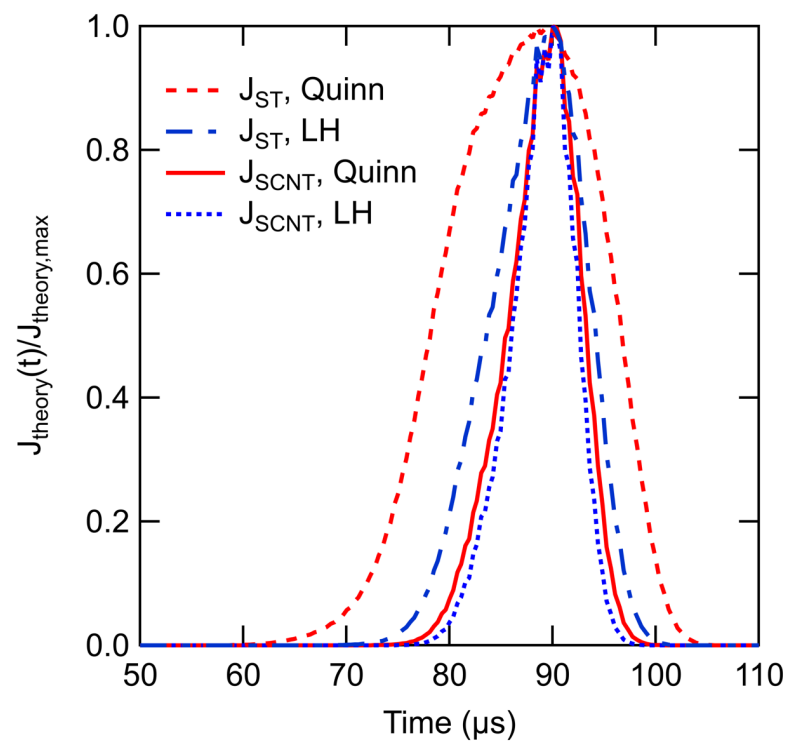

Figure 3. Time-resolved plot of the normalized theoretical nucleation rates, $J_{\text {theory }}(t) / J_{\text {theory,max }}$, for the two nucleation rate expressions $\left(J_{\mathrm{ST}}\right.$ or $\left.J_{\mathrm{SCNT}}\right)$ and the two liquid surface tension correlations (Quinn or LielmeszHerrick, LH). The origin $(t=0)$ corresponds to the throat. The area under the curve defines the characteristic time for nucleation, $\Delta t_{\mathrm{Jmax}}$, and in our earlier work ${ }^{61}$ was generally not a strong function of the nucleation rate expression. Here the uncertainty in physical properties leads to an unusually large difference in the estimated characteristic times - approximately a factor of 2.5 .

For $\mathrm{CO}_{2}$, the values corresponding to conditions at the peak nucleation rate are still well constrained, but uncertainties in the physical properties affect the values of $\Delta t_{J_{\max }}$ more significantly. In particular, Figure 3 shows that for different combinations of theory and surface tension, 3 of the 4 curves yield $\Delta t_{\text {max }}$ values $(7.3,8.50,11.1 \mu \mathrm{s})$ that differ by $\sim 25 \%$ from the average of these three. Although the combination of $J_{\mathrm{ST}}$ and the Quinn surface tension appears to be an outlier, the associated $\Delta t_{\operatorname{Imax}}(18.6 \mu \mathrm{s})$ is still only $\sim 2.5$ times higher than the smallest value. Given the uncertainty in physical properties we have, therefore, chosen to calculate $\Delta t_{\text {Jmax }}$ using $J_{\mathrm{SCNT}}$ and the Quinn parameterization for surface tension, and increased the error bars associated with $J_{\max }$ from our usual factor of 2 , to a factor of 3.

Combining the aerosol number densities, obtained from fits to the SAXS spectra, with the characteristic times obtained from the pressure measurements, we calculate the maximum experimental nucleation rates as

$$
J_{\max }=\frac{N}{\Delta t_{\mathrm{Jmax}}} \times \frac{\rho_{\mathrm{NZ}}}{\rho_{\mathrm{VV}}} .
$$


Here $\rho_{\mathrm{NZ}}$ is the gas density at the location where the nucleation rate is maximized, $\rho_{\mathrm{Vv}}$ is the gas density at the location where $N$ is measured, where the density ratio corrects for the continued expansion of the flow. ${ }^{67}$ In the absence of coagulation, the values of $J_{\max }$ are independent of the measurement location downstream of the nucleation zone.

\section{G. Molecular Dynamics Simulations}

Classical molecular dynamics simulations (MD) were employed to study the nucleation of $\mathrm{CO}_{2}$ clusters from mixtures of $\mathrm{CO}_{2}$ and argon carrier gas, as well as the surface energy of $\mathrm{CO}_{2}$ crystals. $\mathrm{CO}_{2}$ molecules are described by the TraPPE potential, ${ }^{68}$ with parameters that have been fitted to reproduce the experimental liquid-vapor coexistence line. This potential also reproduces the low-temperature vapor-liquid equilibria and solid-vapor equilibria reasonably well. ${ }^{69}$ The molecular geometry of $\mathrm{CO}_{2}$ is rigid with a fixed $\mathrm{C}-\mathrm{O}$ bond length of $1.16 \AA$ and an $\mathrm{O}-\mathrm{C}-\mathrm{O}$ angle of $180^{\circ}$. The rigidity of the model does not significantly affect the phase equilibrium properties compared to a flexible model. ${ }^{70}$ The non-polar Ar atom was modeled with the potential of Hirschfelder et al. ${ }^{71}$ Intermolecular pairwise interactions between atoms $i$ and $j$ with partial charges $q_{i}$ and $q_{j}$ separated by a distance $r_{i j}$ are described by the sum of Lennard-Jones and Coulomb interactions as

$$
U_{i j}=4 \epsilon_{i j}\left[\left(\frac{\sigma_{i j}}{r_{i j}}\right)^{12}-\left(\frac{\sigma_{i j}}{r_{i j}}\right)^{6}\right]+\frac{q_{i} q_{j}}{4 \pi \epsilon_{0} r_{i j}},
$$

where $\epsilon_{i j}$ and $\sigma_{i j}$ denote the Lennard-Jones energy and distance parameters, and $\epsilon_{0}$ is the vacuum permittivity. The force field parameters for $\mathrm{C}, \mathrm{O}$ and $\mathrm{Ar}$ are listed in Table 1. LorentzBerthelot mixing rules are used for interactions between unlike atoms.

Table 1: Atomistic interaction parameters for $\mathrm{CO}_{2}$ and $\mathrm{Ar}$

\begin{tabular}{|l|l|l|l|}
\hline Atom type $i$ & $q_{i}(\mathrm{e})$ & $\epsilon_{i i} / k_{\mathrm{B}}(\mathrm{K})$ & $\sigma_{i i}(\AA)$ \\
\hline $\mathrm{C}$ & +0.70 & 27.0 & 2.80 \\
\hline $\mathrm{O}$ & -0.35 & 79.0 & 3.05 \\
\hline $\mathrm{Ar}$ & 0 & 119.8 & 3.40 \\
\hline
\end{tabular}


We have carried out large-scale direct simulations of nucleation from the vapor phase using $10648 \mathrm{CO}_{2}$ molecules and an equal number of carrier gas atoms, at densities ranging from 0.8 to $1.2 \times 10^{-5} \AA^{-3}$ at $75 \mathrm{~K}$. We employed the LAMMPS MD code ${ }^{72}$, using a Velocity-Verlet integrator and a time step of $5 \mathrm{fs}$. Emerging clusters were identified using the Stillinger criterion for carbon with a radius of $6 \AA$. The standard approach of using a global thermostat is not suitable for directly simulating a nucleating vapor since heat is removed from the nucleating clusters in an unphysical manner. It has been shown that the best way to avoid this artefact is to only thermostat the carrier gas molecules, even though the computational cost is increased significantly. ${ }^{34,35,43}$ Here we applied a Nosé-Hoover thermostat with a time constant equal to 0.1 ps to the Ar atoms only.

To study the phase and structure of the clusters in the nucleation stage, we isolated clusters of size $\geq 10$ molecules from the nucleation simulations and continued to simulate them in vacuum at $75 \mathrm{~K}$ using a Nosé-Hoover thermostat with a rather long time constant of 5 ps to prevent too aggressive thermalization. In these simulations, electrostatic interactions were calculated in real space only, with a cut off exceeding the cluster diameter. The clusters were simulated for at least $50 \mathrm{~ns}$ to compare the final equilibrated structure to the structure during nucleation, in terms of carbon-carbon coordination numbers and radial distribution functions

$$
G(r)=\frac{1}{N_{\mathrm{FCC}}}\left\langle\sum_{i \neq j} \delta\left(r_{i j}-r\right)\right\rangle,
$$

where $N_{\text {FCC }}$ denotes the reference number density in the $\mathrm{FCC} \mathrm{CO}_{2}$ crystal at $T=75 \mathrm{~K}$ and $p=1$ bar.

To study the surface energy, we created FCC crystal slabs exposing two (100), (110), or (111) surfaces, by breaking the periodicity and introducing a vacuum gap along the box in the $z$ direction, and simulated them with MD at constant volume and temperature $T=75 \mathrm{~K}$. The surface energy $E_{\mathrm{s}}$ was calculated as

$$
E_{\mathrm{S}}=\frac{\left\langle E_{\mathrm{p}}\right\rangle-\left\langle E_{\mathrm{p}, \mathrm{bulk}}\right\rangle}{2 A}
$$

where $\left\langle E_{\mathrm{p}}\right\rangle$ and $\left\langle E_{\mathrm{p}, \text { bulk }}\right\rangle$ denote the average potential energy of the system with two interfaces, and the average potential energy of the same number of molecules in the bulk FCC crystal, respectively, and $A$ is the surface area of a single interface. 


\section{EXPERIMENTAL RESULTS AND DISCUSSION}

As summarized in Table 2, pressure trace measurements were performed using $\mathrm{CO}_{2}$ - $\mathrm{Ar}$ gas mixtures ranging from 2 to $39 \mathrm{~mol} \% \mathrm{CO}_{2}$, where these bounds were determined by our ability to control the flow and ensure the nucleation event was complete by the nozzle exit. All expansions started from the same stagnation temperature and pressure, $T_{0}=293.15 \mathrm{~K}$ and $p_{0}=61.15 \pm 0.04$ $\mathrm{kPa}$.

Table 2. Summary of the initial conditions and those corresponding to the maximum nucleation rate. All experiments started from $T_{0}=293.15$ and $p_{0}=61.15 \pm 0.04 \mathrm{kPa}$. The system was comprised of $\mathrm{CO}_{2}$ in $\mathrm{Ar}$ with concentrations ranging from 2.0 to $39.0 \mathrm{~mol} \%$. Here, $p_{\mathrm{v} 0}$ is the initial partial pressure of $\mathrm{CO}_{2}$, and $p_{J \max }, T_{J \max }$, and $S_{J \max }$, are the $\mathrm{CO}_{2}$ partial pressure, flow temperature, and saturation ratio at which the nucleation rates are maximized. The characteristic time $\Delta t t_{\mathrm{Jmax}}$ was calculated using $J_{\mathrm{SCNT}}$ and the Quinn equation for surface tension. The maximum value of $N$ measured in the flow occurs just past the onset of particle formation and is indicated by $N_{\text {onset. }}$ The value of $N$ measured $7 \mathrm{~cm}$ downstream of the throat is denoted by $N(7 \mathrm{~cm})$. These two values of $N$ are used to calculate $J_{\max }$ (onset) and $J_{\max }(7 \mathrm{~cm})$, respectively. A dash indicates SAXS measurements were not made under these conditions. Values in italics have been interpolated.

\begin{tabular}{|c|c|c|c|c|c|c|c|c|c|}
\hline $\mathrm{CO}_{2}$ & $p_{\mathrm{v} 0}$ & $p_{J \max }$ & $T_{J \max }$ & $S_{J \max }$ & $\Delta t_{\max }$ & $N_{\text {onset }}$ & $J_{\max }($ onset $)$ & $N(7 \mathrm{~cm})$ & $J_{\max }(7 \mathrm{~cm})$ \\
\hline $\mathrm{mol} \%$ & $(\mathrm{~Pa})$ & $(\mathrm{Pa})$ & $(\mathrm{K})$ & & $(\mu \mathrm{s})$ & $\left(\mathrm{cm}^{-3}\right)$ & $\left(\mathrm{cm}^{-3} \mathrm{~s}^{-1}\right)$ & $\left(\mathrm{cm}^{-3}\right)$ & $\left(\mathrm{cm}^{-3} \mathrm{~s}^{-1}\right)$ \\
\hline 2.0 & 1200 & 39 & 75.1 & 61000 & 13.2 & - & - & - & - \\
\hline 3.0 & 1800 & 61 & 77.8 & 29000 & 11.2 & $4.42 \mathrm{E}+12$ & $3.94 \mathrm{E}+17$ & $2.42 \mathrm{E}+12$ & $2.87 \mathrm{E}+17$ \\
\hline 3.0 & 1800 & 63 & 78.2 & 25000 & 7.3 & $4.42 \mathrm{E}+12$ & $6.01 \mathrm{E}+17$ & $2.42 \mathrm{E}+12$ & $4.39 \mathrm{E}+17$ \\
\hline 4.0 & 2400 & 87 & 80.2 & 16000 & 6.8 & - & - & - & - \\
\hline 4.5 & 2800 & 110 & 80.5 & 17000 & 6.8 & - & - & $2.19 \mathrm{E}+12$ & $4.29 \mathrm{E}+17$ \\
\hline 5.0 & 3100 & 110 & 81.2 & 13000 & 6.6 & - & - & - & - \\
\hline 6.0 & 3700 & 130 & 82.0 & 12000 & 7.2 & $4.07 \mathrm{E}+12$ & $5.62 \mathrm{E}+17$ & $2.08 \mathrm{E}+12$ & $3.83 \mathrm{E}+17$ \\
\hline 8.00 & 4890 & 176 & 82.1 & 15000 & 7.7 & - & - & - & - \\
\hline 9.00 & 5500 & 198 & 82.5 & 14500 & 6.1 & - & - & $1.92 \mathrm{E}+12$ & $4.25 \mathrm{E}+17$ \\
\hline 10.0 & 6100 & 227 & 83.6 & 11100 & 8.1 & - & - & - & - \\
\hline 12.0 & 7330 & 281 & 86.0 & 5920 & 7.2 & $3.73 \mathrm{E}+12$ & $5.21 \mathrm{E}+17$ & $1.93 \mathrm{E}+12$ & $3.74 \mathrm{E}+17$ \\
\hline 12.0 & 7330 & 278 & 85.7 & 6490 & 7.2 & $3.73 \mathrm{E}+12$ & $5.14 \mathrm{E}+17$ & $1.93 \mathrm{E}+12$ & $3.68 \mathrm{E}+17$ \\
\hline 15.0 & 9160 & 345 & 86.2 & 6790 & 6.8 & - & - & $1.90 \mathrm{E}+12$ & $3.76 \mathrm{E}+17$ \\
\hline 18.0 & 10991 & 411 & 87.2 & 5780 & 6.1 & $3.38 \mathrm{E}+12$ & $5.55 \mathrm{E}+17$ & $1.82 \mathrm{E}+12$ & $4.00 \mathrm{E}+17$ \\
\hline 18.0 & 10991 & 408 & 87.6 & 5030 & 8.5 & $3.38 \mathrm{E}+12$ & $3.98 \mathrm{E}+17$ & $1.82 \mathrm{E}+12$ & $2.85 \mathrm{E}+17$ \\
\hline 21.0 & 12800 & 448 & 88.2 & 4550 & 8.0 & - & - & $1.74 \mathrm{E}+12$ & $2.82 \mathrm{E}+17$ \\
\hline 24.0 & 14700 & 528 & 88.4 & 5020 & 8.8 & $2.73 \mathrm{E}+12$ & $3.09 \mathrm{E}+17$ & $1.70 \mathrm{E}+12$ & $2.31 \mathrm{E}+17$ \\
\hline 27.0 & 16500 & 572 & 89.6 & 3720 & 9.3 & - & - & $1.64 \mathrm{E}+12$ & $2.20 \mathrm{E}+17$ \\
\hline 31.2 & 19100 & 701 & 91.9 & 2270 & 9.8 & $2.27 \mathrm{E}+12$ & $2.31 \mathrm{E}+17$ & $1.57 \mathrm{E}+12$ & $1.86 \mathrm{E}+17$ \\
\hline
\end{tabular}




\begin{tabular}{|l|l|l|l|l|l|c|c|c|c|}
\hline 33.0 & 20200 & 695 & 90.8 & 3130 & 10.7 & - & - & $1.59 \mathrm{E}+12$ & $1.80 \mathrm{E}+17$ \\
\hline 36.0 & 22000 & 757 & 91.4 & 2850 & 11.4 & - & - & $1.51 \mathrm{E}+12$ & $1.59 \mathrm{E}+17$ \\
\hline 38.9 & 23800 & 770 & 91.7 & 2650 & 12.6 & $2.57 \mathrm{E}+12$ & $2.04 \mathrm{E}+17$ & $1.68 \mathrm{E}+12$ & $1.60 \mathrm{E}+17$ \\
\hline 39.0 & 23800 & 782 & 91.9 & 2540 & 12.3 & - & - & $1.48 \mathrm{E}+12$ & $1.43 \mathrm{E}+17$ \\
\hline 39.3 & 24000 & 793 & 92.3 & 2290 & 11.9 & $2.57 \mathrm{E}+12$ & $2.16 \mathrm{E}+17$ & $1.68 \mathrm{E}+12$ & $1.70 \mathrm{E}+17$ \\
\hline
\end{tabular}

\section{A. Onset of nucleation}

Figure 4(a) illustrates selected position-resolved pressure and temperature data for experiments with low (3 mol\%), intermediate (12 mol\%), and high (24 mol\%) $\mathrm{CO}_{2}$

concentrations. The broad range of $\mathrm{CO}_{2}$ concentrations investigated here lead to some interesting phenomena associated with the change in the heat capacity ratio $\gamma$ of the mixture. In particular, as $\gamma$ decreases from 1.66 for $2 \mathrm{~mol}_{\%} \mathrm{CO}_{2}$, to 1.54 for $39 \mathrm{~mol}_{\%} \mathrm{CO}_{2}$, see Fig. 4(b), the expansion softens considerably, and the lowest temperature that can be reached within the nozzle at 39 $\mathrm{mol}^{\%} \mathrm{CO}_{2}$ increases by $\sim 14 \mathrm{~K}$ relative to the $2 \mathrm{~mol} \%$ curve. Consequently, as the concentration of $\mathrm{CO}_{2}$ initially increases, the position of $J_{\max }$ does not move upstream as quickly as expected if $\gamma$ were constant. In fact, as illustrated in Fig. 4(b), once the concentration reaches $12 \mathrm{~mol} \%$, additional $\mathrm{CO}_{2}$ causes the position of $J_{\max }$ to shift downstream again. Eventually, the expansion would no longer reach low enough temperatures within the nozzle to initiate the phase transition.

One of the biggest challenges associated with working at such low temperatures is the potential for contamination. According to Heiler, ${ }^{73}$ solid particle number densities of at least $10^{8}$ $\mathrm{cm}^{-3}$ are required to interfere with homogeneous nucleation, and introducing such a dense aerosol from outside the nozzle is difficult in our system. Rather, particle formation within the nozzle, stemming from trace amounts of condensable vapors with lower vapor pressure (i.e. water, alcohol, alkanes), is a more likely potential source of seeds for heterogeneous nucleation of $\mathrm{CO}_{2}$. Contamination was identified as illustrated in Fig. S-1 of the Supplementary Information, where repeat experiments for the same starting conditions found onset temperatures and pressures separated by up to $3.7 \mathrm{~K}$ and $23 \mathrm{~Pa}$, respectively, and saturation ratios differing by up to two orders of magnitude. The most consistent data were generated by first purging the system with argon for 1 hour at 90 SLM before starting a set of measurements, and this is the protocol used for all of the results presented here. 

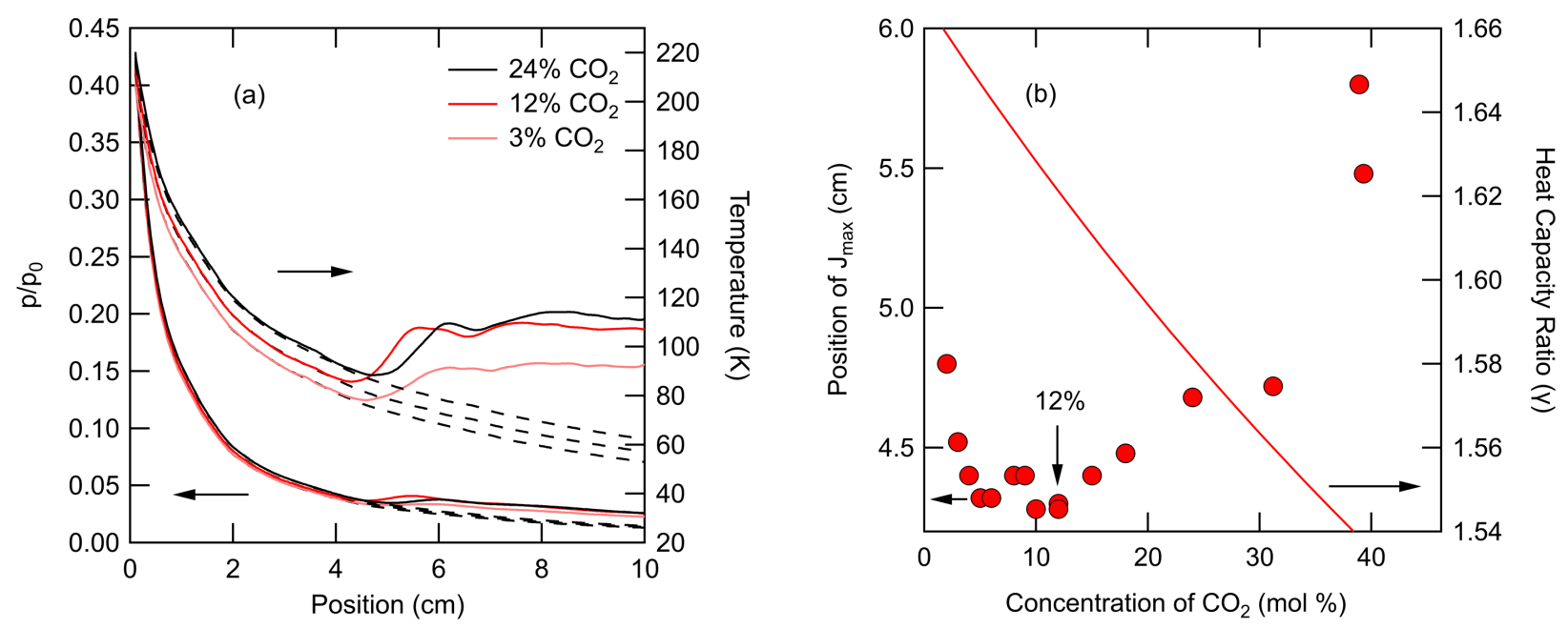

Figure 4. (a) Pressure and temperature traces for (top to bottom) high, intermediate, and low concentrations of $\mathrm{CO}_{2}$ in Ar. Dashed lines are the isentropic expansions expected in the absence of a phase transition. Solid lines are the measured expansions with condensation. (b) The position of $J_{\max }$ is not a monotonic function of the $\mathrm{CO}_{2}$ concentration because the decreasing heat capacity ratio softens the expansion as the $\mathrm{CO}_{2}$ concentration increases.

The $\mathrm{CO}_{2}$ partial pressures and temperatures corresponding to $J_{\max }$ are illustrated in the Volmer plot, Fig. 5(a). Overall, our experiments cover a range in partial pressures from 38.6 to $793 \mathrm{~Pa}$, and corresponding temperatures from 75.1 to $92.3 \mathrm{~K}$. The data exhibit a linear relationship when $\log \left(p_{J_{\max }}\right)$ is plotted against $1 / T_{J_{\max }}$. This is consistent with the findings of Volmer ${ }^{74}$ who established that data with a constant nucleation barrier should fall on a gently curving line when plotted on $\log (p)$ vs $1 / T$. Over a limited temperature range, this should look like a straight line. Similar behavior is also observed for the alkanes, alcohols, and water. ${ }^{24,67,75 \text {, }}$ 76

As shown in the Volmer plot (Fig. 5(a)) and the phase diagram (Fig. 5(b)), our data agree with the two other research groups that have studied the homogeneous condensation of $\mathrm{CO}_{2}$ in supersonic nozzles ${ }^{11,14}$ across a surprisingly wide range of pressures and temperatures. This suggests that free energy barriers associated with nucleation for these three sets of experiments are likely quite comparable. The free jet data of Ramos et al. ${ }^{77,78}$ are also very consistent, where the higher pressures in the free jet data reflect the higher cooling rates that make it possible to probe the metastable region more deeply and, thereby, further reduce the associated free energy barrier. For the onset measurements, the presence or absence of a carrier gas does not appear to be important. This observation is consistent with our earlier $\mathrm{Ar}^{79}$ and $\mathrm{N}_{2}{ }^{80}$ measurements where 

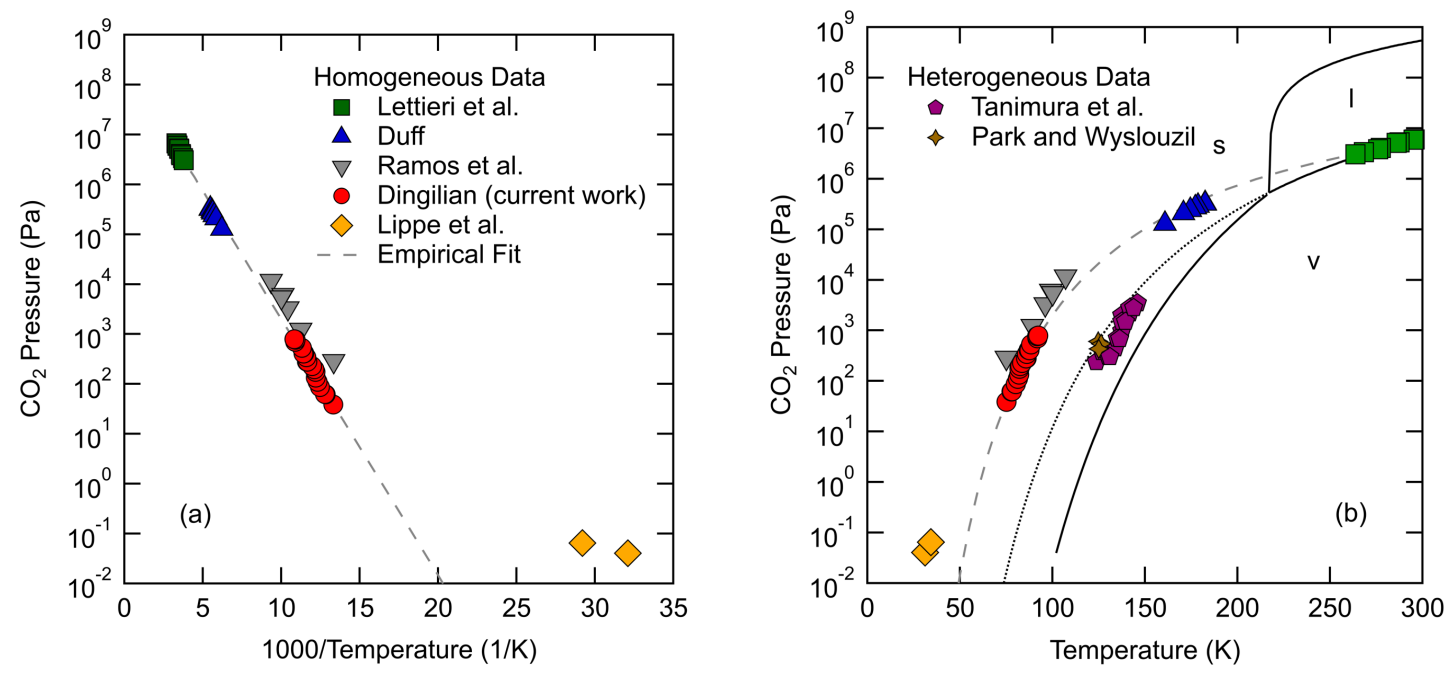

Figure 5. (a) The combined Volmer plot suggests that free energy barriers are similar for all of the supersonic nozzle measurements ${ }^{11,14}$ except for those of Lippe et al. ${ }^{28}$ The free jet data lie at higher pressures consistent with the significantly higher cooling rates associated with those experiments. ${ }^{77,78}$ (b) The measured temperatures and pressures at $J_{\max }$ corresponding to the onset of homogeneous and heterogeneous ${ }^{10,32}$ nucleation of $\mathrm{CO}_{2}$ in supersonic nozzles are shown on the phase diagram of $\mathrm{CO}_{2}$. Solid lines correspond to equilibrium phase boundaries. The dotted line is the extrapolated liquid-vapor pressure curve. The dashed line is the empirical fit from (a). The symbols are the same as in (a).

onset data measured in the presence of a carrier gas transitioned smoothly to those measured for the pure vapor.

The data from Lippe et al., ${ }^{28}$ at an estimated nucleation rate of $10^{15} \mathrm{~cm}^{-3} \mathrm{~s}^{-1}$, lie at significantly lower temperatures and pressures. Although the $\mathrm{CO}_{2}$ partial pressures in the gas phase are much lower in their experiments than in ours, the extremely low temperatures still lead to more extreme saturation ratios - on the order of $10^{50}$ to $10^{60}$ - before clusters start to form and grow. ${ }^{28}$ It may be that nucleation in their experiments approaches the kinetic limit, and, if so, it would be unrealistic to expect the onset data to follow the same trend as phase transitions under more moderate conditions that are controlled by a free energy barrier. On the phase diagram, Fig. 5(b), with the exception of the Lettieri et al. ${ }^{11}$ data, the homogeneous $\mathrm{CO}_{2}$ data all lie to the left of the vapor-solid and the extrapolated vapor-liquid equilibrium lines (dotted line) and are also well-separated from the heterogeneous $\mathrm{CO}_{2}$ nucleation data of Tanimura et al. ${ }^{10}$ and Park and Wyslouzil ${ }^{32}$. Although the phase of the final condensate in the current experiments differs from that of Lettieri et al., i.e. solid particles rather than liquid droplets, it is uncertain if it differs from that of Duff. The consistency in the trend in onset data between the experiments, however, 
suggests that the initial critical clusters that control particle formation may be similar across all nucleation conditions, i.e. the critical clusters may all be liquid like. This idea is also supported by the Raman spectra measured by Ramos et al., ${ }^{77}$ where the feature associated with clusters containing $\sim 150$ molecules was assigned to a liquid-like state. In contrast, the spectra for larger clusters exhibited features consistent with the crystalline solid.

\section{B. Particle size and number density}

SAXS measurements were made both as a function of position and at a fixed position, 7.0 $\mathrm{cm}$ downstream of the physical throat. Fixed position experiments are a more efficient way to measure nucleation rates, and at this position nucleation was completely quenched for all of the experimental conditions investigated. Figure 6(a) shows typical raw background, sample, and background subtracted SAXS spectra as well as the fit to the background subtracted spectrum. The fit assumes that the $\mathrm{X}$-rays scatter from an aerosol consisting of a collection of polydisperse spheres that follow a Schulz distribution. Except for the smallest particles, the fit parameters are not sensitive to the shape of the underlying size distribution (lognormal, Schultz, or Gaussian) ${ }^{61}$ Figure 6(b) shows the measured number average particle radius and the aerosol number densities as a function of the concentration of $\mathrm{CO}_{2}$ entering the system. At $7.0 \mathrm{~cm}$ downstream of the physical throat, particles ranged from 4.0 to $6.7 \mathrm{~nm}$ in radius. As the concentration of $\mathrm{CO}_{2}$ increases, the average particle radius increases rapidly both because fewer particles are made (decrease in number density as seen in Fig. 6(b)) and because there is more material available to condense. At a fixed position, the flow warms as the heat capacity ratio softens the expansion. Thus, above a certain concentration, particles form further and further downstream, and eventually the particle sizes at $7.0 \mathrm{~cm}$ decrease because condensational growth is not yet complete. 

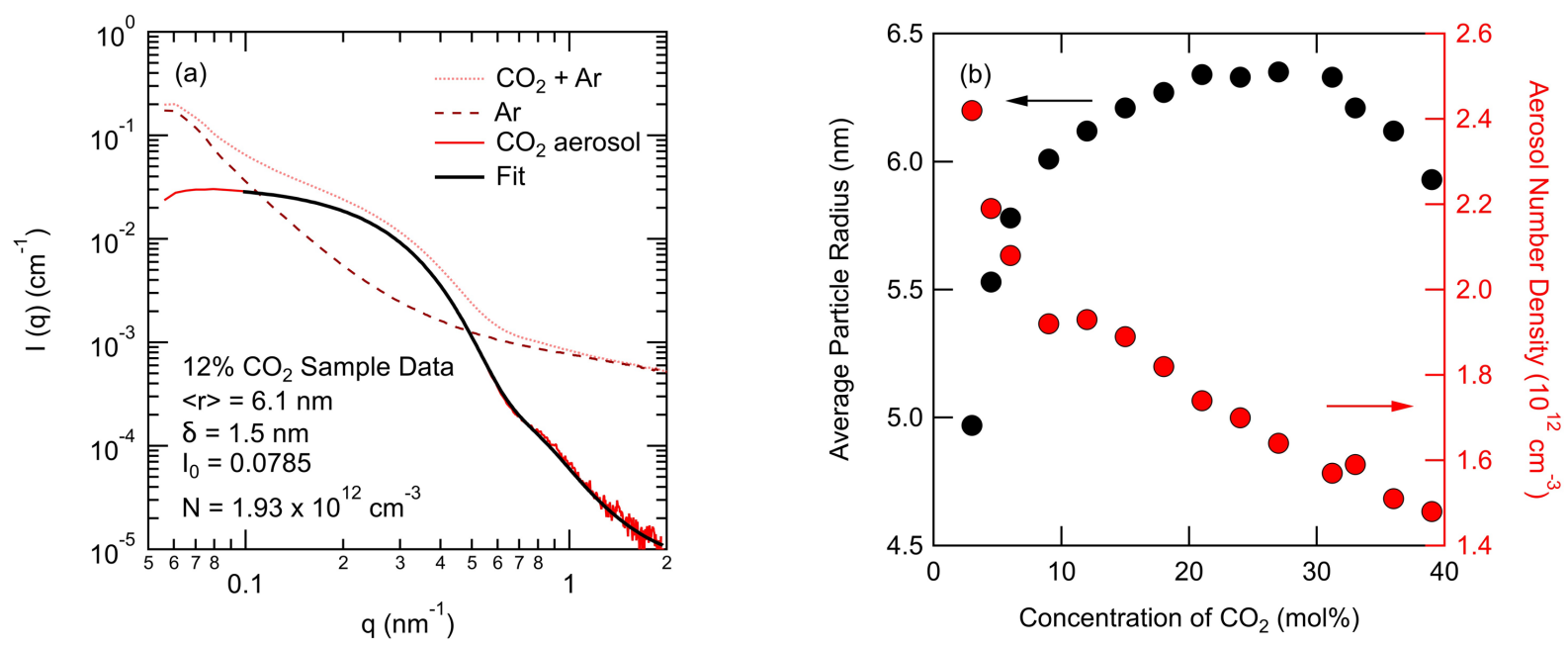

Figure 6. (a) SAXS spectra as a function of the scattering vector $\boldsymbol{q}$ corresponding to the background (Ar), the sample $\left(\mathrm{CO}_{2}+\mathrm{Ar}\right)$, and the background subtracted scattering stemming from the $\mathrm{CO}_{2}$ aerosol. The bold line is the best fit assuming scattering from a Schultz polydisperse distribution of spheres, and it agrees well with the measured aerosol spectrum. (b) The average particle radius and aerosol number density as a function of $\mathrm{CO}_{2}$ concentration measured $7.0 \mathrm{~cm}$ downstream of the throat in nozzle T3_mica.

The decrease in number density with increasing $\mathrm{CO}_{2}$ concentration can be explained by the coupling between nucleation, particle growth, and changes in the expansion rate. Initially, as the partial pressure of $\mathrm{CO}_{2}$ increases, particles that nucleate can grow more rapidly, releasing heat to the flow and shutting off nucleation more rapidly. The characteristic times decrease accordingly - up to concentrations of $12 \mathrm{~mol} \%$. Further increases in the $\mathrm{CO}_{2}$ concentration are now associated with expansions characterized by weaker temperature gradients. Less rapid changes in temperature make it possible for the phase transition to be initiated at lower nucleation rates.$^{61}$ Although the characteristic times increase, nucleation rates decrease slightly more quickly leading to a net decrease in particle concentration.

Position-resolved SAXS measurements were made for 7 systems containing $3-39 \mathrm{~mol} \%$ $\mathrm{CO}_{2}$ in Ar. Figure 7(a) shows position-resolved average particle radii for the same three concentrations of $\mathrm{CO}_{2}$ illustrated in Fig. 4(a). Figure 7(b) displays the corresponding specific, aerosol number densities, $N^{\prime}=N / \rho$, i.e. the aerosol number densities normalized by the gas 

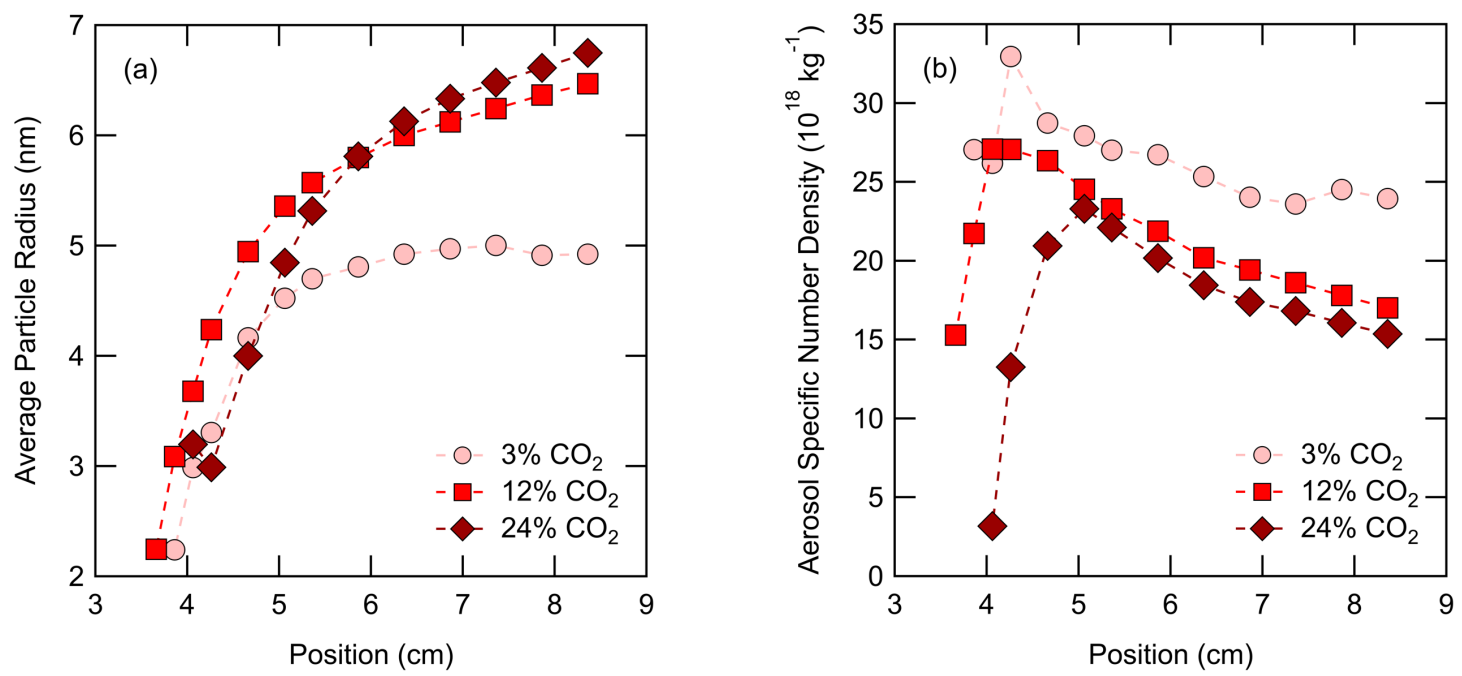

Figure 7. (a) Position-resolved particle radii for 3, 12, and $24 \mathrm{~mol} \% \mathrm{CO}_{2}$. (b) Position-resolved specific number density data. The continuous increase in particle size coupled with a decreases in specific number densities indicate the $\mathrm{CO}_{2}$ particles are coagulating.

density to account for the continued expansion of the flow. The remaining position resolved measurements are shown in Figs. S-2(a) and S-2(b) of the Supplementary Information. Initially, specific aerosol number density and average particle size both increase as nucleation and condensational particle growth occur simultaneously. Once heat release quenches the nucleation event, no new particles are formed, and the specific aerosol number density drops with a continued but slower increase in particle size. At this point, coagulation becomes an important mode of particle growth. For most $\mathrm{CO}_{2}$ concentrations, particles are still growing at the exit of the nozzle. For the $3 \mathrm{~mol} \% \mathrm{CO}_{2}$ data, we observe a similar drop in specific aerosol number density, but the particle radius does not increase to the same extent.

Although all SAXS experiments used the same purging procedure before starting the measurements, directly comparing the SAXS results to the PTM data revealed a slight inconsistency between the two. On average, we observed scattering signals from SAXS measurements about 0.5 to $0.7 \mathrm{~cm}$ upstream of the position of $J_{\max }$ calculated from the PTM data. This is illustrated in Fig. 8, where the solid red curves are the theoretical normalized nucleation rates, i.e. $J_{\text {theory }}(x) / J_{\text {theory,max }}$ that show the expected progress of the nucleation event from start to finish. The dashed gray curves are the expected normalized specific aerosol number densities, $N^{\prime} / N_{\max }^{\prime}$ derived from the nucleation rate calculations in the absence of coagulation. Particles 
are expected to appear slightly before the position of the maximum nucleation rate and in a manner such that the measured specific aerosol number density data (filled circles) overlap with the predicted specific aerosol number densities (dashed line). Instead, particles are first observed significantly earlier.
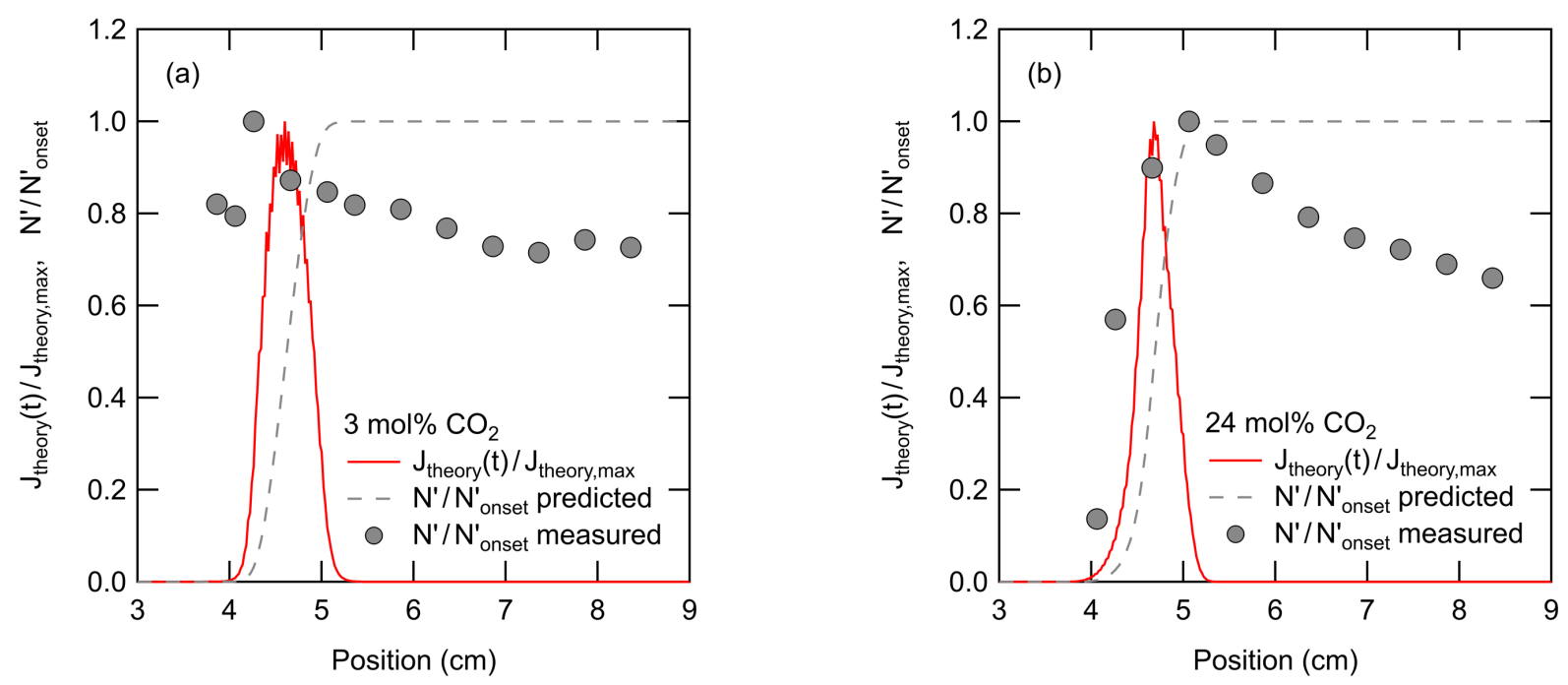

Figure 8. Comparison of SAXS and PTM data for (a) $3 \mathrm{~mol} \% \mathrm{CO}_{2}$ and (b) $24 \mathrm{~mol} \% \mathrm{CO}_{2}$. The solid curves correspond to the normalized nucleation rates and the gray dashed lines indicate the corresponding normalized specific aerosol number densities. The circles are the measured data. In both cases particles appeared earlier in the SAXS experiments than expected based on the PTMs.

We do not believe this inconsistency implies heterogeneous nucleation for the following reasons. First, for the SAXS measurements the system was purged using the same protocol used for the PTM measurements. Second, as illustrated in Fig. 6(a), the scattering spectra of the particles were well fit assuming a distribution of polydisperse spheres. This contrasts with SAXS spectra from Tanimura et al.'s heterogeneous nucleation experiments ${ }^{10}$ of $\mathrm{CO}_{2}$ on $\mathrm{H}_{2} \mathrm{O}$, where the data could not be easily fit to scattering from spherical particles. Third, based on the homogeneous water nucleation rate data of Amaya and Wyslouzil, ${ }^{81}$ we can estimate the temperatures at which water particles should form if $\mathrm{CO}_{2}$ contains the maximum allowed water contamination. As illustrated in Figure S-3, if water particles formed, they should have been available for heterogeneous nucleation well upstream of the expected heterogeneous nucleation boundary established by Tanimura et al. ${ }^{10}$ Thus, if heterogeneous nucleation on water ice were important, scattering should have been observed much further upstream than where it was first 
detected in our SAXS experiments. Similar arguments apply to alkanes ${ }^{82}$ or other contaminant molecules that condense much earlier than $\mathrm{CO}_{2}$ under the same expansion conditions. Finally, we estimated the temperatures and pressures corresponding to $J_{\max }$ based on the positions where particles first appeared in SAXS. As illustrated in Fig. S-3, these points lie very close to the $J_{\max }$ data calculated from PTM and are still well-separated from the heterogeneous $\mathrm{CO}_{2}$ nucleation curve. This suggests our data do correspond to homogeneous nucleation or the nearhomogeneous limit.

Another possibility is that of ion-induced nucleation. During SAXS measurements, the high-energy x-ray beam can ionize the gas phase molecules within the nozzle. If this occurs in the region just upstream of the expected location of $J_{\max }$, the ions are exposed to extremely high $\mathrm{CO}_{2}$ saturation ratios that could promote heterogeneous nucleation of $\mathrm{CO}_{2}$ onto the ions. Ionization should not, however, interfere with homogeneous nucleation below a threshold saturation ratio. Thus, aerosol number densities measured further downstream - at much lower saturation ratios, should be due to homogeneous nucleation alone.

\section{Experimental nucleation rates}

Experimental nucleation rates were calculated as described in Sec. III.F. The trend in $\Delta t_{\text {Jmax }}$ follows that displayed by the position at which the nucleation rate is maximized. In particular, the characteristic times decrease as nucleation moves further upstream and increases as nucleation moves downstream again. As the concentration of $\mathrm{CO}_{2}$ increases, the expansion softens and less rapid expansions are associated with longer characteristic times. ${ }^{61}$

As summarized in Fig. 9, $\mathrm{CO}_{2}$ experimental nucleation rates range from $\sim 1.5 \times 10^{17} \mathrm{~cm}^{-}$ ${ }^{3} \mathrm{~s}^{-1}$ to $\sim 6 \times 10^{17} \mathrm{~cm}^{-3} \mathrm{~s}^{-1}$. The good agreement between rates based on the fixed position measurements $\left(J_{\max }(7 \mathrm{~cm})\right)$ and those based on the position resolved measurements $\left(J_{\max }(\right.$ onset $\left.)\right)$, confirms that our results are relatively insensitive to the location of the aerosol number density measurement. The rates based on the fixed-position data are generally slightly lower, consistent with coagulation decreasing the specific aerosol number densities by up to $45 \%$ as the flow moves downstream. Nevertheless, the overlapping error bars demonstrate that the values still agree to within our stated accuracy of a factor of 3 . The rates measured here are also consistent with previous nucleation measurements in supersonic nozzles, ${ }^{24,67,75,76}$ that range from approximately $10^{16} \mathrm{~cm}^{-3} \mathrm{~s}^{-1}$ to $10^{18} \mathrm{~cm}^{-3} \mathrm{~s}^{-1}$. 


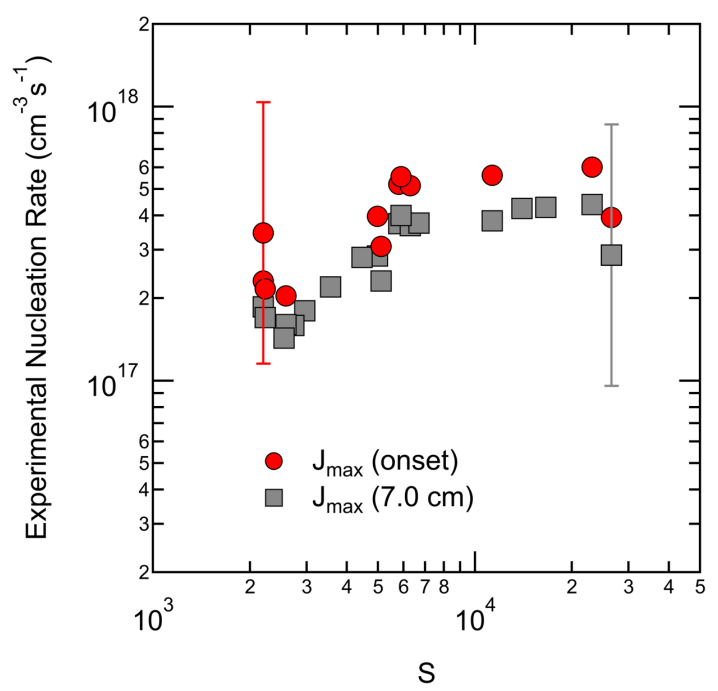

Figure 9. Experimental nucleation rates calculated using the values of $N_{\text {onset }}$ and $N(7.0 \mathrm{~cm})$ agree within the stated factor of 3 uncertainty. The latter are lower due to coagulation of the aerosol between the nucleation and measurement zones.

\section{IR spectra of $\mathrm{CO}_{2}$}

The Fourier transform infrared (FTIR) spectroscopy measurements made for $12 \mathrm{~mol} \%$ $\mathrm{CO}_{2}$ in $\mathrm{Ar}$ are shown as a function of position in Fig. 10(a). In Fig. 10(b) the contributions from the gas phase have been subtracted from the full spectra to better observe the condensed phase, and the particle sizes based on SAXS measurements are also noted. For positions up to $3.2 \mathrm{~cm}$ from the throat, only the gas phase ${ }^{12} \mathrm{CO}_{2}$ band centered at $2350 \mathrm{~cm}^{-1}$ is observed. As particles form and grow, a condensed phase peak centered at $2359 \mathrm{~cm}^{-1}$ with a shoulder near $2370 \mathrm{~cm}^{-1}$ appears $\sim 3.6 \mathrm{~cm}$ downstream of the throat and grows in intensity with particle size. Comparing our data to spectra measured by Isenor et al. ${ }^{45}$ suggest that the condensate consists of octahedral cubes of solid $\mathrm{CO}_{2}$ rather than spheres. 

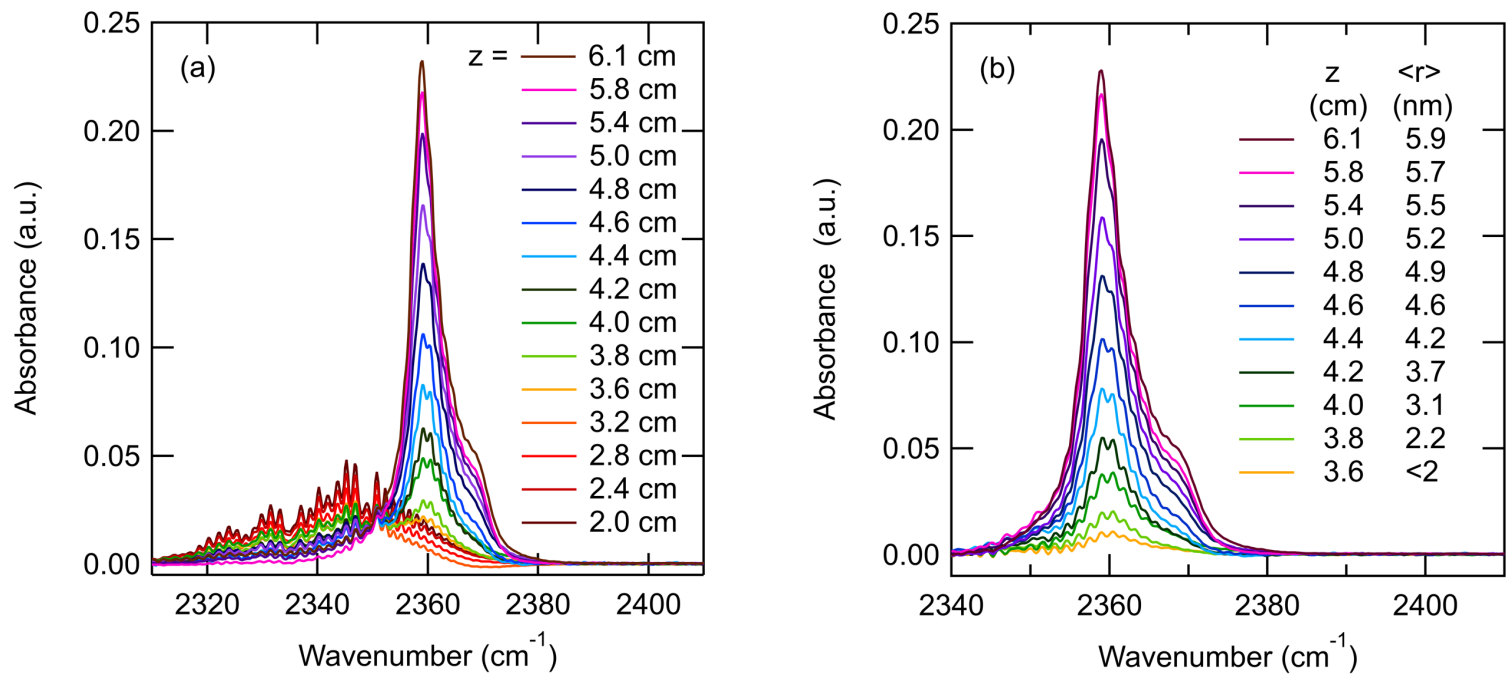

Figure 10. (a) The FTIR spectra measured $2.0 \mathrm{~cm}$ to $6.1 \mathrm{~cm}$ downstream of the throat. The spectrum with the highest intensity corresponds to $6.1 \mathrm{~cm}$. (b) The condensed phase spectra after the gas phase contribution has been empirically subtracted. The particle sizes are based on interpolating SAXS measurements.

\section{COMPARISONS WITH CLASSICAL NUCLEATION AND SCALING THEORIES}

\section{A. The structure of the nucleating clusters}

Comparing experimental results with the predictions of any nucleation theory is often hampered by the lack of appropriate physical property data in the experimentally relevant temperature range. For the highly supercooled conditions investigated here, where temperatures are $125-142 \mathrm{~K}$ below the triple point, even the nature of the critical cluster, i.e. whether it is crystalline, liquid, or amorphous, is uncertain. Of these three possibilities, the formation of a crystalline cluster directly from the vapor phase is highly unlikely since the cost of forming an organized cluster directly from the vapor is generally higher than forming a disorganized liquid cluster. Furthermore, in recent large scale MD simulations of Lennard-Jones fluid, ${ }^{83}$ nucleation from the vapor phase always occurred via the supercooled liquid, even at temperatures $T / T_{\mathrm{c}}$ of $\sim 0.23$ where $T_{\mathrm{c}}$ is the critical temperature. Post-critical clusters only froze when they reached sizes greater than approximately 800 molecules. Likewise, in $\mathrm{CO}_{2}$ free jet experiments, Ramos et al. ${ }^{77}$ assigned the first band that appeared in the Raman scattering spectrum of the condensed phase to a liquid-like phase.

In our FTIR experiments we were not able to observe a change in the spectra that indicated a transition in the condensate from the liquid to a solid, in part because of strong 
interference from the gas phase spectra. Thus, we turned to MD simulations to examine the nature of the nucleating clusters. In addition to examining the clusters generated during the nucleation process, we also examined the structure they adopted after equilibration when isolated from the surrounding vapor. In both cases, we calculated the carbon-carbon radial distribution function and the coordination numbers for 436 clusters containing between 10 and 109 molecules and averaged the results by size ranges. As illustrated in Fig. 11, even though these relatively small clusters exhibit a clear crystalline structure in equilibrium at $75 \mathrm{~K}$, the clusters remain in a liquid or amorphous phase during the nucleation period as the latent heat released by growth events is not removed at a high enough rate through collisions with the carrier gas. Although the saturation ratios in the simulated system are about ten times higher than in the experiments, the amount of heat removed from a cluster between growth events should be more or less equal since the ratio of condensable to carrier gas is quite similar in both cases. The latent heat released during a liquid to solid transition would require additional thermalization by the carrier gas. Thus, it is likely that the characteristics of the clusters nucleating at the experimental rate of $10^{17} \mathrm{~cm}^{-3} \mathrm{~s}^{-1}$ are similar to the ones observed in the simulations.
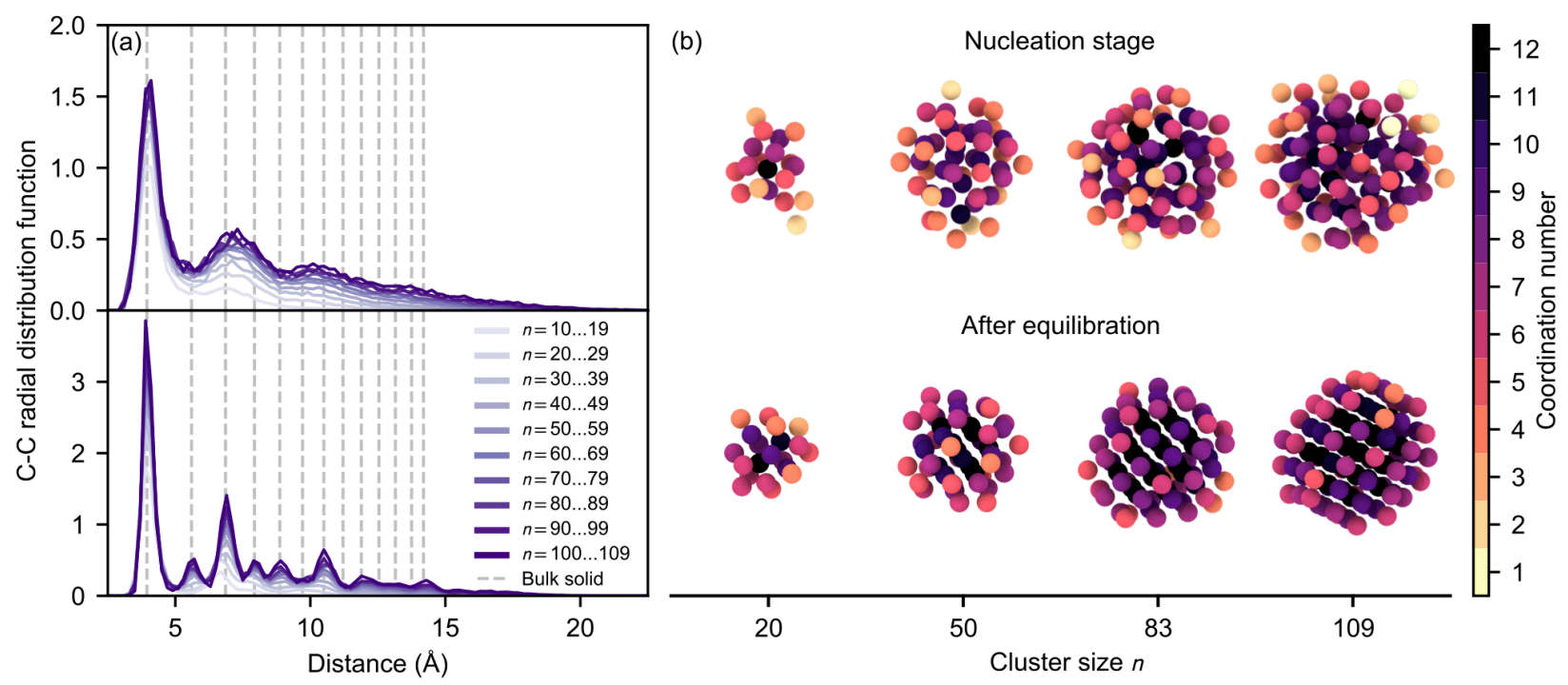

Figure 11 (a) Average C-C radial distribution functions for different post-critical $\mathrm{CO}_{2}$ cluster sizes during a nucleation simulation (top) and after the clusters were isolated and equilibrated at $T=75 \mathrm{~K}$ (bottom). The positions of the peaks in the bulk FCC crystal structure of $\mathrm{CO}_{2}$ at $T=75 \mathrm{~K}$ and $p=1$ bar are indicated by dashed gray lines. (b) Example structures of four different-sized clusters during nucleation (top) and after equilibration (bottom). For clarity, only the molecules' carbon atoms are shown, colored according to their coordination number. 


\section{B. Comparisons with nucleation theory}

For direct comparison between the predictions of theory and experimental nucleation rates, both the state of the critical cluster and the effects of thermalization are important. Thus, all of the comparisons with theory assume liquid like critical clusters that are not fully thermalized by the carrier gas. For the $\mathrm{CO}_{2}$-Ar mixtures used in these experiments, at the conditions corresponding to the maximum nucleation rates, the values of the non-isothermal correction factors vary between 0.015 and 0.17 with the strongest corrections corresponding to the highest $\mathrm{CO}_{2}$ concentrations (Table $\left.\mathbf{S}-\mathbf{1}\right)$.

In the context of the experiments, we are first interested in determining the vapor pressure corresponding to an experimental nucleation rate of $5 \times 10^{17} \mathrm{~cm}^{-3} \mathrm{~s}^{-1}$ as a function of temperature. To do so, we numerically solve for the pressure corresponding to this rate, assuming an average constant non-isothermal factor $f=0.05$, where the latter is within a factor of $\sim 3$ for all of the values of presented in Table S-1. Figure 12 compares the available supersonic nozzle data with the computed pressure values as a function of temperature for all combinations of the two nucleation rate expressions and two surface tensions correlations considered. On the scale of this figure, all of the theories do reasonably well predicting the pressure/temperature pairs for the $\mathrm{CO}_{2}$ condensation experiments of Duff and Lettieri et al. For the current data, the predictions of self-consistent nucleation theory $J_{\mathrm{SCNT}}$ yields onset pressure above the data for either surface tension expression, whereas, simulation-based theory, $J_{\mathrm{ST}}$, yields onset pressures that are slightly below the experimental data for either surface tension expression. None of the theories reproduce the experimentally obtained temperature dependency over the entire temperature range. 


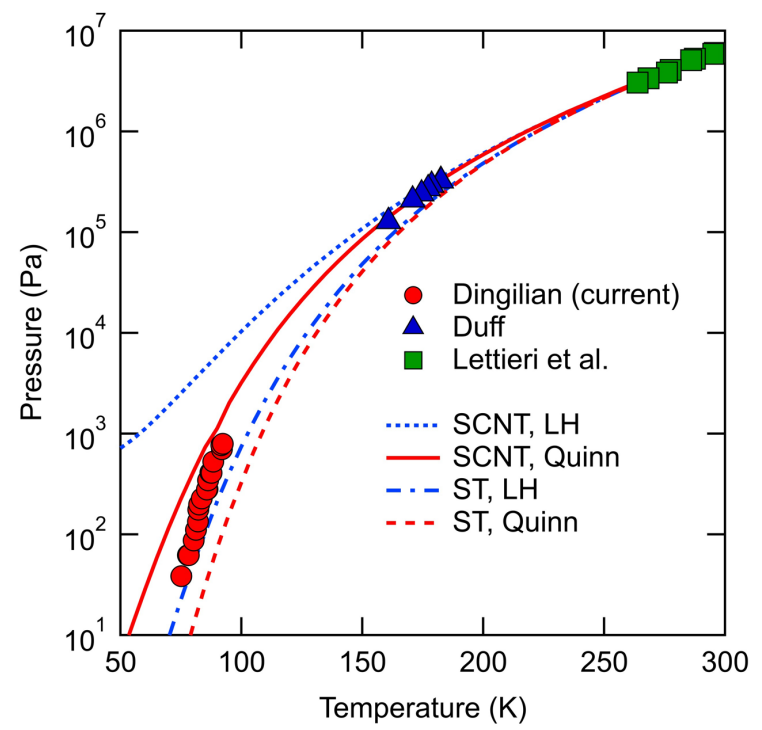

Figure 12. Curves corresponding to a constant non-isothermal nucleation rate of $5 \times 10^{17} \mathrm{~cm}^{-3} \mathrm{~s}^{-1}$ for all combinations of nucleation theory ( $J_{\mathrm{SCNT}}$ or $J_{\mathrm{ST}}$ ) and surface tension correlation (Quinn or Lielmesz-Herrick, $\mathrm{LH}$ ) are compared to the experimental onset of nucleation data of Lettieri, Duff, and the current work.

A more stringent comparison of the data with the two theories is illustrated in Figure 13 where we directly examine the ratio of $J_{\text {exp }} / J_{\text {theory }}$ using both surface tension expressions and $J_{\mathrm{SCNT}}$ or $J_{\mathrm{ST}}$ to calculate the theoretical values. All theoretical values were corrected for non-

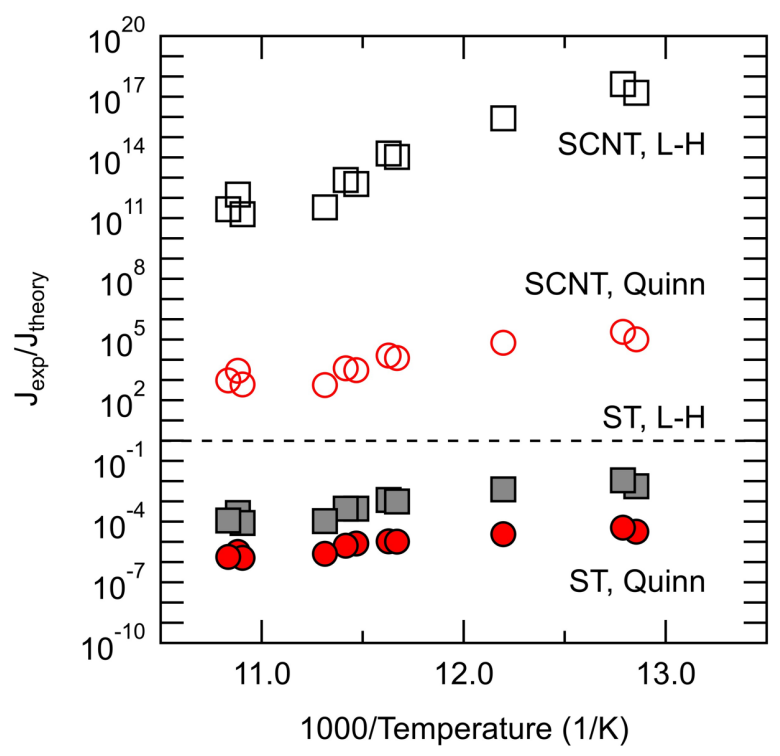

Figure 13. Comparisons between experimental nucleation rates and those calculated using self-consistent classical nucleation theory (SCNT) and simulation based theory (ST) for the surface tension correlations of Quinn or based on the Lielmezs-Herrick (L-H) equation. Non-isothermal corrections are included in both theories. 
isothermal effects based on the experimental conditions at nucleation. Although none of the theory/surface tension combinations is quantitatively correct, the temperature dependence of the ST theory matches that of the experiments slightly better than SCNT does.

Finally, an alternate way to test the consistency of all of the available data, and to predict nucleation rates empirically, is to consider the scaling laws first proposed by Hale et al. ${ }^{84} \mathrm{As}$ illustrated in Figure 14, all of the available data measured in supersonic nozzles are bounded by the $J_{\exp }=10^{17} \mathrm{~cm}^{-3} \mathrm{~s}^{-1}$ and $J_{\exp }=10^{19} \mathrm{~cm}^{-3} \mathrm{~s}^{-1}$ straight lines for an effective omega parameter of $\Omega_{\text {eff }}=1.70$. The value of $\Omega_{\text {eff }}$ is about $25 \%$ lower than the value of $\Omega_{\text {theory }}=2.22$ calculated using

$$
k N_{A}^{2 / 3} \Omega \approx K_{\mathrm{E}}=\left(\mu_{v} / \rho_{l}\right)^{2 / 3} \sigma /\left(T_{c}-T\right)
$$

where $N_{\mathrm{A}}$ is Avogadro's number, $K_{\mathrm{E}}$ is the Eötvös constant and the extrapolated surface tension is that of Quinn. The deviation observed here is comparable to that observed for other small molecules we have studied, i.e. for Ar we found $\Omega_{\text {eff }}: \Omega_{\text {theory }}=1.5: 2$ and for $\mathrm{N}_{2}, \Omega_{\text {eff }}: \Omega_{\text {theory }}=$ 1.54: 2.07. Assuming a solid critical cluster yields $\Omega_{\text {theory }}=3.28$, a value that implies a much greater deviation between the effective and theoretical values of $\Omega$. Although this is not proof that the critical clusters are liquid-like, the difference between $\Omega_{\mathrm{eff}}$ and $\Omega_{\text {theory }}$ is much larger than we have ever observed.

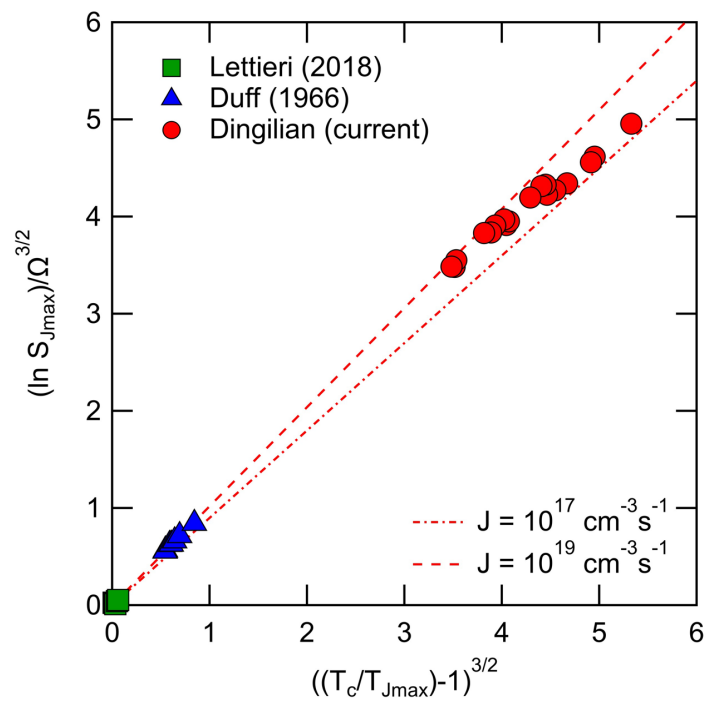

Figure 14. In the Hale plot, the experimental supersonic nozzle data are bounded by the lines corresponding to the nucleation rates $J=10^{19} \mathrm{~cm}^{-3} \mathrm{~s}^{-1}$ and $J=10^{17} \mathrm{~cm}^{-3} \mathrm{~s}^{-1}$ when the effective omega parameter is $\Omega=1.70$. 


\section{SUMMARY AND CONCLUSIONS}

We have measured homogeneous nucleation rates for $\mathrm{CO}_{2}$ by expanding $\mathrm{CO}_{2}$-Ar gas mixtures in a supersonic nozzle where cooling rates are on the order of $0.5 \mathrm{~K} / \mu \mathrm{s}$ near the onset of condensation. The formation and evolution of the resulting aerosol was followed using pressure trace measurements, SAXS, and FTIR spectroscopy for $\mathrm{CO}_{2}$ concentrations between 2.0 and $39.3 \mathrm{~mol} \%$. These conditions led to onset of nucleation characterized by temperature/pressure pairs ranging from $(75 \mathrm{~K}, 39 \mathrm{~Pa})$ to $(92 \mathrm{~K}, 793 \mathrm{~Pa})$ and nucleation rates on the order of $5 \times 10^{17}$ $\mathrm{cm}^{-3} \mathrm{~s}^{-1}$. One surprising observation was the excellent agreement in the onset conditions between the current work and experiments conducted under much higher pressures and temperatures even when conditions approached the critical point. This suggest that the free energy barriers required to initiate the phase transition in the nozzle experiments are must be quite similar. Agreement with free jet expansions experiments ${ }^{77,78}$ was also very good. The higher pressures observed in the free jet are consistent with the significantly higher cooling rates that enable this experiment to probe the metastable region more deeply. Uncertainty in the physical property values - and their temperature dependence - led to greater differences in estimates for the characteristic time than we have observed in the past, but experimental nucleation rates are still quantified to within a factor of 3. Fourier transform infrared (FTIR) spectroscopy are consistent with the aerosol being comprised of crystalline $\mathrm{CO}_{2}$ particles that are cubic solids with either sharp or rounded corners.

Interpreting the experiments was challenging given the high degree of supercooling with respect to any condensed phase. Free jet experiment at comparable temperatures, suggest clusters containing $\sim 150$ molecules are liquid-like, ${ }^{77,78}$ as did MD simulations at comparable temperatures. MD studies also confirmed the importance of thermalization of the critical clusters, and the need to incorporate a size dependent curvature term into the free energy expression. Experimental nucleation rates were therefore compared to the predictions of non-isothermal nucleation theory assuming a liquid like critical cluster. None of the combinations yielded quantitative predictions, although the simulation based theory exhibited a slightly better temperature dependence than SCNT.

For the purposes of modeling $\mathrm{CO}_{2}$ condensation in supersonic flows for CCS applications, the current work and our earlier heterogeneous ${ }^{10,32}$ nucleation experiments both 
demonstrate that high $\mathrm{CO}_{2}$ saturation ratios are required to initiate condensation. For models that incorporate heterogeneous nucleation, assuming that condensation occurs along the extrapolated vapor-liquid equilibrium line, rather than the vapor-solid equilibrium line, appears to be a

reasonable approximation. For those models that assume homogeneous nucleation dominates the phase transition, using the Hale scaling approach with an effective omega parameter of $\Omega=1.70$ appears to be a convenient and fairly robust way to estimate the nucleation rate.

A more detailed presentation of the simulation work, that also explores the best way to extrapolate to the experimental data, will appear in a forthcoming publication.

\section{ACKNOWLEDGEMENTS}

This work was completed with the financial support of the National Science Foundation under grant number CBET-1511498, University of Helsinki, Faculty of Science ATMATH project, and ERC grant no. 692891-DAMOCLES. Supercomputing resources were provided by the CSC-IT Center for Science, Ltd., Finland, and the Finnish Grid and Cloud Infrastructure (urn:nbn:fi:research-infras-2016072533) at the University of Helsinki. This research used resources of the Advanced Photon Source, a U.S. Department of Energy (DOE) Office of Science User Facility operated for the DOE Office of Science by Argonne National Laboratory under Contract No. DE-AC02-06CH11357. We also thank Andrew Amaya, Kehinde Ogunronbi, Kilho Lee, Yensil Park, Martina Lippe, Soenke Seifert, and Randall Winans for their help in conducting the SAXS experiments. We also thank Lahari Pallerla for helping with the FTIR data analysis. 


\section{APPENDIX}

Table A-1: Physical Properties of Ar and $\mathrm{CO}_{2}$

\begin{tabular}{|l|l|}
\hline Argon (Ar) \\
\hline Molecular weight (g/mol) & 39.948 \\
\hline Heat capacity ratio & 1.667 \\
\hline
\end{tabular}

\begin{tabular}{|c|c|}
\hline \multicolumn{2}{|l|}{ Carbon Dioxide $\left(\mathrm{CO}_{2}\right)$} \\
\hline Molecular weight (g/mol) & 44.01 \\
\hline Critical temperature $\boldsymbol{T}_{\mathrm{c}}(\mathrm{K})$ & 304.1282 \\
\hline Critical pressure $\boldsymbol{p}_{\mathrm{c}}(\mathrm{MPa})$ & 7.3773 \\
\hline Triple point & $216.55 \mathrm{~K}, 0.5168 \mathrm{MPa}$ \\
\hline Critical density $\left(\mathrm{kg} / \mathrm{m}^{3}\right)$ & 467.6 \\
\hline $\begin{array}{l}\text { Heat capacity at constant } \mathrm{P} \\
(\mathrm{J} / \mathrm{mol} \cdot \mathrm{K})^{10}\end{array}$ & $25.9162+\left(2.93005 \times 10^{-2}\right) T+\left(2.3825 \times 10^{-5}\right) T^{2}$ \\
\hline $\begin{array}{l}\text { Liquid surface tension, Quinn }{ }^{54} \\
(\mathrm{mN} / \mathrm{m})\end{array}$ & $0.0653(31.35-(T-273.15))^{1.24}$ \\
\hline $\begin{array}{l}\text { Liquid surface tension, fit to } \\
\text { Lielmezs-Herrick equation } \\
(\mathrm{mN} / \mathrm{m})\end{array}$ & $\begin{array}{l}1.004 \sigma_{m} \frac{T}{T_{m}}\left[\frac{\left(T_{c}-T\right) T_{m}}{\left(T_{c}-T_{m}\right) T}\right]^{1.21} \\
\sigma_{\mathrm{m}}=16.9 ; T_{\mathrm{m}}=216.6 \mathrm{~K}\end{array}$ \\
\hline $\begin{array}{l}\text { Liquid density, Span and } \\
\text { Wagner } \\
\left(\rho^{\prime}[=] \mathrm{kg} / \mathrm{m}^{3}\right)\end{array}$ & $\begin{array}{l}\ln \left(\frac{\rho^{\prime}}{\rho_{c}}\right)=\sum_{i=1}^{4} a_{i}\left(1-\frac{T}{T_{c}}\right)^{t_{i}} \\
\mathrm{a}_{1}=1.9245108 ; \mathrm{a}_{2}=-0.62385555 ; \mathrm{a}_{3}=-0.32731127 ; \mathrm{a}_{4}= \\
0.39245142 ; \mathrm{t}_{1}=0.34 ; \mathrm{t}_{2}=0.5 ; \mathrm{t}_{3}=10 / 6 ; \mathrm{t}_{4}=11 / 6\end{array}$ \\
\hline $\begin{array}{l}\text { Equilibrium vapor pressure, } \\
\text { liquid }^{85}(\mathrm{~Pa})\end{array}$ & $101325 * 10^{-\frac{1353}{T}-8.143 \log T+0.006259 T+24.619}$ \\
\hline Heat of vaporization $(\mathrm{kJ} / \mathrm{mol})$ & $\begin{array}{l}28.4363-0.021174 \mathrm{~T}+\left(1.19722 \times 10^{-4}\right) \mathrm{T}^{2}-(4.64865 \times \\
\left.10^{-7}\right) \mathrm{T}^{3}\end{array}$ \\
\hline
\end{tabular}




\section{REFERENCES}

1. J. Gibbins and H. Chalmers, Energy Policy, 2008, 36, 4317-4322.

2. A. Hart and N. Gnanendran, Energy Procedia, 2009, 1, 10.

3. R. S. Haszeldine, Science, 2009, 325, 1647-1652.

4. J. C. M. Pires, F. G. Martins, M. C. M. Alvim-Ferraz and M. Simoes, Chemical Engineering Research and Design, 2011, 89, 1446-1460.

5. P. Markewitz, W. Kuckshinrichs, W. Leitner, J. Linssen, P. Zapp, R. Bongartz, A. Schreiber and T. E. Muller, Energy \& Environmental Science, 2012, 5, 7281-7305.

6. P. Luis, Desalination, 2016, 380, 93-99.

7. H.-B. Xie, C. Li, N. He, C. Wang, S. Zhang and J. Chen, Environmental Science \& Technology, 2014, 48, 1700-1706.

8. N. Borduas, J. P. D. Abbatt and J. G. Murphy, Environmental Science \& Technology, 2013, 47, 6377-6383.

9. H.-B. Xie, J. Elm, R. Halonen, N. Myllys, T. Kurten, M. Kulmala and H. Vehkamaki, Environmental Science \& Technology, 2017, 51, 8422-8431.

10. S. Tanimura, Y. Park, A. Amaya, V. Modak and B. E. Wyslouzil, RSC Advances, 2015, 5, 105537-105550.

11. C. Lettieri, D. Paxson, Z. Spakovszky and P. Bryanston-Cross, Journal of Engineering for Gas Turbines and Power, 2018, 140, 041701.

12. A. Hernandez Nogales, M.S. Thesis, Escola Tècnica Superior d'Enginyeria Industrial de Barcelona, 2011.

13. R. Ahmad Samawe, K. Rostani, A. Mohd Jalil, M. Esa and N. Othman, presented in part at the Offshore Technology Conference Kuala Lumpur, Malaysia, 2014.

14. K. M. Duff, Ph.D. Thesis, Massachusetts Institute of Technology, 1966.

15. F. L. Daum, AIAA Journal, 1963, 1, 1043-1046.

16. J. I. Yellott, Transaction of ASME, 1934, 56, 411-430.

17. C. Wen and Y. Yang, presented in part at the 79th European Association of Geoscientists \& Engineers Conference and Exhibition, 2017.

18. F. T. Okimoto and J. M. Brouwer, presented in part at the Gas Processors Association, San Antonio, 2003.

19. J. M. Brouwer and H. D. Epsom, presented in part at the Offshore Europe, Aberdeen, UK, 2003.

20. A. Borissov, G. Mirzoev, and V. Shtern, United States Pat. 8, 790,455 B2, 2014, 1-17.

21. V. Alfyorov, L. Bagirov, L. Dmitriev, V. Feygin, S. Imayev and J. R. Lacey, Oil \& Gas Journal, 2005, 53-58.

22. R. A. Altam, T. A. Lemma and S. R. Jufar, presented in part at the MATEC Web of Conferences, 2017.

23. M. Haghighi, K. A. Hawboldt and M. A. Abdi, Journal of Natural Gas Science and Engineering, 2015, 27, 109-121.

24. B. E. Wyslouzil and J. Wolk, The Journal of Chemical Physics, 2016, 145, 211702.

25. J. Diemand, R. Angelil, K. K. Tanaka and H. Tanaka, The Journal of Chemical Physics, 2013, 139, 074309.

26. S. Toxvaerd, The Journal of Chemical Physics, 2001, 115, 8913-8920. 
27. M. Hammer, P. E. Wahl, R. Anantharaman, D. Berstad and K. Y. Lervag, Energy Procedia, 2014, 63, 243-252.

28. M. Lippe, U. Szczepaniak, G.-L. Hou, S. Chakrabarty, J. J. Ferreiro, E. Chasovskikh and R. Signorell, The Journal of Physical Chemistry A, 2019, 123, 2426-2437.

29. A. M. Binnie and J. R. Green, Proceedings of the Royal Society A, 1941, 181, 134-154.

30. I. Faro, T. R. Small and F. K. Hill, Journal of Applied Physics, 1952, 23, 40-43.

31. P. P. Wegener, Progress in Astronautics and Rocketry, 1964, 15, 701-724.

32. Y. Park and B. E. Wyslouzil, Physical Chemistry and Chemical Physics, 2019, 21, 82958313.

33. J. Wedekind and R. Strey, Journal of Chemical Physics, 2007, 126, 134103.

34. K. Yasuoka and M. Matsumoto, Journal of Chemical Physics, 1998, 109, 8451-8462.

35. R. Halonen, E. Zapadinsky and H. Vehkamäki, Journal of Chemical Physics, 2018, 148, 164508.

36. K. K. Tanaka, A. Kawano and H. Tanaka, Journal of Chemical Physics, 2014, 140, 114302.

37. R. Angélil, J. Diemand, K. K. Tanaka and H. Tanaka, Journal of Chemical Physics, 2015, 143, 064507.

38. M. Duška, T. Němec, J. Hrubý, V. Vinš and B. Planková, EPJ Web of Conferences, 2015, 92, 02013.

39. S. Braun, V. Kalikmanov and T. Kraska, Journal of Chemical Physics, 2014, 140, 124305.

40. F. Römer and T. Kraska, Journal of Chemical Physics, 2007, 127, 234509.

41. M. Horsch, J. Vrabec, M. Bernreuther, S. Grottel, G. Reina, A. Wix, K. Schaber and H. Hasse, Journal of Chemical Physics, 2008, 128, 164510.

42. K. Yasuoka and M. Matsumoto, Journal of Chemical Physics, 1998, 109, 8463-8470.

43. J. Wedekind, D. Reguera and R. Strey, Journal of Chemical Physics, 2007, 127, 064501.

44. S. Ayuba, D. Suh, K. Nomura, T. Ebisuzaki and K. Yasuoka, Journal of Chemical Physics, 2018, 149, 044504.

45. M. Isenor, R. Escribano, T. C. Preston and R. Signorell, Icarus, 2013, 223, 591-601.

46. W. G. Courtney, Journal of Chemical Physics, 1961, 35, 2249-2250.

47. S. L. Girshick and C.-P. Chiu, Journal of Chemical Physics, 1990, 93, 1273-1277.

48. D. Saltz, Journal of Chemical Physics, 1994, 101, 6038-6051.

49. J. Feder, K. C. Russell, J. Lothe and G. M. Pound, Advances in Physics, 1966, 15, 111-178.

50. R. C. Tolman, Journal of Chemical Physics, 1949, 17, 333-337.

51. Y. A. Lei, T. Bykov, S. Yoo and X. C. Zeng, Journal of the American Chemical Society, 2005, 127, 15346-15347.

52. N. Bruot and F. Caupin, Physical Review Letters, 2016, 116, 056102.

53. K. K. Tanaka, J. Diemand, R. Angelil and H. Tanaka, Journal of Chemical Physics, 2014, 140, 194310.

54. E. L. Quinn, Journal of the American Chemical Society, 1927, 49, 2704-2711.

55. J. Lielmezs and T. A. Herrick, Thermochimica Acta, 1989, 141, 113-124.

56. S. E. Wood, Ph.D. Thesis, University of California, Los Angeles, 1999.

57. G. N. Muratov and V. P. Skripov, Teplofizika Vysokikh Temperatur, 1982, 20, 596-598.

58. R. Span and W. Wagner, Journal of Physical and Chemical Reference Data, 1996, 25, 1509-1596. 
59. W. Duschek, R. Kleinrahm and W. Wagner, The Journal of Chemical Thermodynamics, 1990, 22, 827-840.

60. T. P. Mangan, C. G. Salzmann, J. M. C. Plane and B. J. Murray, Icarus, 2017, 294, 201-208.

61. Y. J. Kim, B. E. Wyslouzil, G. Wilemski, J. Wolk and R. Strey, The Journal of Physical Chemistry A, 2004, 108, 4365-4377.

62. B. E. Wyslouzil, C. H. Heath, J. L. Cheung and G. Wilemski, The Journal of Chemical Physics, 2000, 113, 7317-7329.

63. S. Seifert, R. E. Winans, D. M. Tiede and P. Thiyagarajan, Journal of Applied Crystallography, 2000, 33, 782-784.

64. A. Manka, H. Pathak, S. Tanimura, J. Wolk, R. Strey and B. E. Wyslouzil, Physical Chemistry and Chemical Physics, 2012, 14, 4505-4516.

65. M. Kotlarchyk and S.-H. Chen, The Journal of Chemical Physics, 1983, 79, 2461-2469.

66. P.J. Linstrom and W.G. Mallard, Eds., NIST Chemistry WebBook, NIST Standard Reference Database Number 69, National Institute of Standards and Technology, Gaithersburg MD, 20899, https://doi.org/10.18434/T4D303.

67. K. Mullick, A. Bhabhe, A. Manka, J. Wolk, R. Strey and B. E. Wyslouzil, The Journal of Physical Chemistry B, 2015, 119, 9009-9019.

68. J. J. Potoff and J. I. Siepmann, AlChE Journal, 2001, 47, 1676-1682.

69. B. Chen, J. I. Siepmann and M. L. Klein, Journal of Physical Chemistry B, 2001, 105, 98409848.

70. J. G. Harris and K. H. Yung, Journal of Physical Chemistry, 1995, 99, 12021-12024.

71. J. O. Hirschfelder, C. F. Curtiss and R. B. Bird, Molecular theory of gases and liquids, Wiley, New York, 1954.

72. S. Plimpton, Journal of Computational Physics, 1995, 117, 1-19.

73. M. Heiler, Ph.D. Thesis, Universitat Karlsruhe, 1999.

74. M. Volmer, Kinetik der Phasenbildung, Dresden u. Leipzig, 1939.

75. D. Ghosh, D. Bergmann, R. Schwering, J. Wölk, R. Strey, S. Tanimura and B. E. Wyslouzil, Journal of Chemical Physics, 2010, 132, 024307.

76. K. E. Ogunronbi, A. Sepehri, B. Chen and B. E. Wyslouzil, Journal of Chemical Physics, 2018, 148, 144312.

77. A. Ramos, J. M. Fernández, G. Tejeda and S. Montero, Physical Review A, 2005, 72, 053204.

78. A. Ramos, G. Tejeda, J. M. Fernández and S. Montero, AIP Conference Proceedings, 2012, 1501, 1383-1389.

79. S. Sinha, A. Bhabhe, H. Laksmono, J. Wölk, R. Strey and B. Wyslouzil, The Journal of Chemical Physics, 2010, 132, 064304.

80. A. Bhabhe and B. Wyslouzil, The Journal of Chemical Physics, 2011, 135, 244311.

81. A. J. Amaya and B. E. Wyslouzil, The Journal of Chemical Physics, 2018, 148, 084501.

82. K. E. Ogunronbi and B. E. Wyslouzil, Journal of Chemical Physics, 2019, 151, 154307.

83. K. K. Tanaka, J. Diemand, H. Tanaka and R. Angélil, Physical Review E, 2017, 96, 022804.

84. B. N. Hale, Physical Review A, 1986, 33, 4156-4163.

85. A. Michels, T. Wassenaar, T. Zwietering and P. Smits, Physica, 1950, 16, 501-504. 